\title{
A Role for Receptor Protein Tyrosine Phosphatase $\lambda$ in Midbrain Development
}

\author{
Anja Badde and Dorothea Schulte \\ Department of Neuroanatomy, Max Planck Institute for Brain Research, 60528 Frankfurt, Germany
}

The mid-hindbrain boundary (MHB) harbors an important organizing center for the adjacent brain regions. Here, we present evidence that the receptor protein tyrosine phosphatase $\lambda(R P T P \lambda)$ is part of the complex molecular network that maintains and shapes the MHB region. RPTP $\lambda$ is expressed in a tight band of cells in the caudal midbrain, anterior to the transverse ring of Wnt 1 expression. Forced expression of $R P T P \lambda$ across the mid-hindbrain region repressed expression of Wnt1, whereas RNA interference-mediated knock-down of $R P T P \lambda$ resulted in expansion and distortion of the Wnt1 domain. When ectopically expressed in the mesencephalon, $R P T P \lambda$ specifically inhibited the induction of Wnt1 expression after subsequent stimulation with F $8 f$. Reduced Wnt1 expression after RPTP $\lambda$ transfection correlated with a decrease in Ras- mitogen-activated protein kinase activity at the MHB. We further show that in the embryonic midbrain, RPTP $\lambda$ can bind to $\beta$-catenin, a central component of the canonical Wnt signaling pathway. Overexpression of $R P T P \lambda$ suppressed the activity of a $\beta$-catenin responsive promoter in the midbrain and reduced progenitor cell proliferation. Cotransfection of Wnt 1 or of a stabilized form of $\beta$-catenin together with $R P T P \lambda$ partially rescued the $R P T P \lambda$-mediated proliferation defect. Together, these data suggest that RPTP $\lambda$ may play a dual role in the control of midbrain development: as a negative modulator of Fgf8-induced Wnt 1 expression at the MHB, which may help to confine the Wnt1 domain to it characteristic tight ring at the MHB; and as an inhibitor of canonical Wnt signaling through interaction with and presumably sequestration of $\beta$-catenin.

Key words: RPTP $\psi ; F g f ;$ Wnt1; $\beta$-catenin; midbrain; chick

\section{Introduction}

The mid-hindbrain (MHB) (isthmic) organizer, located near the constriction between the developing mesencephalic and metencephalic vesicles, acts as a secondary organizer for the control of growth and pattern formation of the adjacent midbrain and hindbrain (Nakamura et al., 1986; Martinez and AlvaradoMallart, 1990; Martinez et al., 1991). Its activity is regulated by an interdependent network of nuclear factors and signaling proteins, which includes the transcription factors En1/En2, Pax2/ Pax5, Lmx1b, Hes1/Hes3, irol/iro7, and members of the Fof and Wnt families of secreted proteins (Bally-Cuif et al., 1992; Rowitch and McMahon, 1995; Araki and Nakamura, 1999; Shamim et al., 1999; Adams et al., 2000; Hirata et al., 2001; Itoh et al., 2002; Matsunaga et al., 2002; O'Hara et al., 2005).

Fibroblast growth factor 8 ( Fgf8), expressed in the rostral hindbrain adjacent to the MHB, is of central importance for midbrain

\footnotetext{
Received July 4, 2007; revised March 21, 2008; accepted April 17, 2008.

This work was supported by the International Max-Planck Research School Program in Structure and Function of Biological Membranes (Frankfurt, Germany) (A.B.). We thank C. Tabin (Harvard Medical School, Boston, MA), C. Logan (University of Calgary, Calgary, Alberta, Canada), C. Krull (University of Michigan, Ann Arbor, MI), H. Rohrer (Max-Planck Institute for Brain Research, Frankfurt, Germany), F. Costantini (Columbia University Medical Center, New York, NY), and R. Dorsky (University of Utah, Salt Lake (ity, UT) for reagents; C. Ziegler for excellent technica assistance; G. O'Sullivan for critically reading this manuscript; and A. Vennemann, E. Dohle, and A. Poplawski for experimental help.

Correspondence should be addressed to Dorothea Schulte, Department of Neuroanatomy, Max Planck Institute for Brain Research, Deutschordenstrasse 46, 60528 Frankfurt, Germany. E-mail: schulte@mpih-frankfurt.mpg.de.

A. Badde's present address: Helmholtz Zentrum München, German Research Center for Environmental Health, Institute of Developmental Genetics, Ingolstädter Landstrasse 1, D-85764 Neuherberg, Munich, Germany. DOI:10.1523/JNEUROSCI.5593-07.2008

Copyright $\odot 2008$ Society for Neuroscience $\quad 0270-6474 / 08 / 286152-13 \$ 15.00 / 0$
}

and hindbrain development. Beads soaked in recombinant Fgf8 and implanted in prosomeres $\mathrm{p} 1, \mathrm{p} 2$ of the diencephalon, in the midbrain or hindbrain can mimic the inductive capacity of isthmic transplants (Crossley et al., 1996; Martinez et al., 1999; Shamim et al., 1999; Irving and Mason, 2000). Conversely, zebrafish ace mutants, which lack functional $F g f$, and mice hypomorphic for $F g f 8$ fail to maintain gene expression at the MHB and display massive defects in midbrain and cerebellar development (Meyers et al., 1998; Reifers et al., 1998). An important target of Fgf8 at the MHB is the secreted glycoprotein Wnt1. After isthmic organizer formation, Wnt1 expression is restricted to a narrow band of cells in the caudal mesencephalon, anterior to the Fgf8 expression domain (Bally-Cuif et al., 1995; Hollyday et al., 1995). Wht signaling is critically involved in mid-hindbrain development, because targeted deletion or tissue-specific overexpression of Wnt1 at the MHB lead to massive perturbation of midhindbrain growth (Thomas and Capecchi, 1990; McMahon et al., 1992; Panhuysen et al., 2004).

Reversible protein phosphorylation on tyrosine residues is a key mechanism underlying intracellular signal transduction. Protein tyrosine phosphatases (PTPs) antagonize the activity of protein tyrosine kinases (PTKs) and thereby limit the duration and intensity of the PTK signal (Stoker and Dutta, 1998; den Hertog, 1999; Paul and Lombroso, 2003; Tonks, 2006). Receptor protein tyrosine phosphatase $\lambda(R P T P \lambda$; also called $R P T P \psi)$, a member of the type IIb family of receptor tyrosine phosphatases, is expressed in a spatially and temporally dynamic manner in the embryo and has been shown recently to be necessary for somito- 
genesis and convergent extension during gastrulation (Aerne et al., 2003; Aerne and Ish-Horowicz, 2004). We reported previously the developmental expression of RPTP $\lambda$ in the CNS and have shown that its expression is particularly dynamic anterior to the isthmic constriction (Badde et al., 2005). Here, we have taken a gain-of-function and loss-of-function approach to show that $R P T P \lambda$ can modulate mesencephalic development through a dual mechanism: it inhibits the induction of Wnt1 expression by $F g f 8$, which may help to restrict the Wnt1 domain to its characteristic tight ring at the $\mathrm{MHB}$, and binds to $\beta$-catenin and appears to thereby negatively modulate canonical Wnt signaling.

\section{Materials and Methods}

Expression constructs and in ovo electroporation. cDNAs encoding fulllength mouse RPTP $\lambda$ [National Center for Biotechnology Information (NCBI) accession number U55057] or RPTP $\lambda-\Delta I C$ (corresponding to nucleotides 352-2701 of NCBI accession number U55057) were fused to a triple HA-tag and cloned into the expression vector pMES, which includes an internal ribosomal entry site (IRES)-green fluorescent protein (GFP) cassette (Swartz et al., 2001) or into the vector pMIWIII, which lacks IRES-GFP (Suemori et al., 1990). The coding sequence of chick $R P T P \lambda$ was cloned using degenerate primers based on published sequences of the mouse, rat, and human orthologs and found to be mostly identical to NCBI accession number AY147868 (sequences are available on request). The coding sequences of chick $F g f b b$, mouse $W n t 1$, or mouse En1 were cloned into pMIWIII. For $d n R a s^{N 17}$-pMES, a dominantnegative form of the Ras protein, which contains a serine to asparagine mutation at residue 17, was inserted into pMES (Clontech). Axin-pMES carried the full-length coding region of chick Axin, a generous gift from F. Costantini (Columbia University Medical Center, New York, NY) (Zeng et al., 1997). For caß-catenin-pMIWIII, a stabilized form of $\beta$-catenin was cloned into pMIWIII (Funayama et al., 1995). For the rescue experiment with $R P T P \lambda$ and Wnt1, the coding sequence of GFP in $R P T P \lambda$-pMES was replaced by a single ClaI site (RPTP $\lambda$-pMEC). The coding region of Wnt1 was then cloned into this ClaI site resulting in $R P T P \lambda$-Wnt1-pMEC. Unless otherwise noted, $1-2 \mu \mathrm{g} / \mu \mathrm{l}$ of each construct were injected into the neural tube of Hamburger-Hamilton stage 9-10 (HH9-HH10) chick embryos (White Leghorn) (Hamburger and Hamilton, 1992). Electroporation was performed as described, except that five pulses of $10 \mathrm{~V}$ for $50 \mathrm{~ms}$ were given to facilitate DNA uptake (Schulte et al., 1999). To visualize DNA uptake, $0.5-0.8 \mu \mathrm{g} / \mu \mathrm{l}$ of plasmids expressing enhanced GFP (GFP-pMIWIII), Discosoma red fluorescent protein (dsRed-pCMV), or a nuclear version of red fluorescent protein ( $n R F P$-pCAGGS) were coelectroporated. To control for possible unspecific effects of the procedure, pMES (which carries IRES-GFP) or GFPpMIWIII was introduced. For consecutive electroporation into the same region of the neural tube (see Fig. 3A), GFP with or without the fulllength form of $R P T P \lambda$ or the catalytically inactive allele $R P T P \lambda-\triangle I C$ were introduced into the mesencephalic vesicle at HH9-10. Six hours after initial transfection, a time window known to be sufficient for robust transgene expression (Momose et al., 1999), an expression plasmid carrying the coding sequence of $F g f b b$ together with a plasmid carrying $d s R e d$ were electroporated into the same area that had received the first electroporation $6 \mathrm{~h}$ earlier. Only embryos that exhibited extensive coexpression of both fluorescent markers, indicating that both transgenes were targeted to the same region of the neural tube, were chosen for additional analysis. For TOP:dgfp reporter analysis, $1 \mu \mathrm{g} / \mu \mathrm{l}$ of the TOP: dgfp reporter construct, which drives expression of destabilized green fluorescent protein off the TOPFLASH promoter/enhancer (Korinek et al., 1997; Dorsky et al., 2002), were electroporated into the mesencephalic vesicle alone or in combination with $1.5 \mu \mathrm{g} / \mu \mathrm{l} R P T P \lambda$-pMIWIII or $1.5 \mu \mathrm{g} / \mu \mathrm{l} R P T P \lambda-\Delta I C$-pMIWIII. GFP expression was monitored and documented $24 \mathrm{~h}$ later.

Bead implantation. Heparin-coated acrylic beads (Sigma-Aldrich) were soaked in $0.015-0.15 \mathrm{mg} / \mathrm{ml}$ mouse recombinant Fgf8b protein (R\&D Systems) overnight at $4^{\circ} \mathrm{C}$, washed in sterile PBS, and split with forceps. Appropriately sized bead fragments were implanted into the lateral wall of the mesencephalic vesicle of HH11 chick embryos. The embryos were collected $24 \mathrm{~h}$ after bead implantation and processed for in situ hybridization.

RNA in situ hybridization. To ensure specificity of the probe, a partial sequence of chick $R P T P \lambda$ was used for all in situ hybridization experiments (Badde et al., 2005). RNA probes for $c G r g 4, c S p r o u t y 2$, and $c S e f$ were cloned from chick HH15-20 whole head total RNA by reverse transcriptase-PCR with gene-specific primers (primer sequences are available on request). The $\mathrm{cDNAs}$ used to generate in situ probes for $c E n 1$, $c F g f 8, c \operatorname{Lmx} 1 b, c \mathrm{Otx} 2, c \mathrm{Pax} 2, c \mathrm{Pax} 5$, or $c W n t 1$ were gifts from C. Tabin (Harvard Medical School, Boston, MA), C. Logan (University of Calgary, Calgary, Alberta, Canada), or H. Rohrer (Max Planck Institute for Brain Research, Frankfurt, Germany). In situ hybridization on whole embryos was performed as described previously (Schulte et al., 1999). For open book preparation, the mesencephalic vesicle was opened along the dorsal midline and flat-mounted. Two-color in situ hybridization was performed as described with the exception that the first transcripts were detected using 5-bromo-4-chlor-indolyl-phosphate (BCIP) and nitroblue-tetrazolium-chloride as chromophores, which results in a dark blue/purple precipitate, whereas the second transcripts were detected with BCIP alone, which results in a bright blue/turquoise precipitate (Schulte and Cepko, 2000). Specimens were photographed with a Zeiss StemiSV11 microscope and a Canon PowerShot G5 camera or with a Leica MZ12 microscope and a Leica MPS60 camera. Brightness and contrast were adjusted in Adobe Photoshop (Adobe Systems). In Figure 1, C, $D, D^{\prime}, I^{\prime}$, and $I^{\prime \prime}$ were false colored in Adobe Photoshop for better visual contrast.

Immunohistochemical staining. Localization of ectopically expressed $R P T P \lambda-H A$ or $R P T P \lambda-\triangle I C-H A$ was visualized on cryosections using a polyclonal anti-HA antibody (1:1000; Roche Diagnostics). GFP expression was confirmed using a polyclonal antibody (1:1000; Invitrogen). The mitotic index was determined with a polyclonal anti-phosphorylated Histone H3 (pH3) antibody (1:1000; Upstate Biotechnology). All antibodies were diluted in PBS containing 5\% Chemiblocker (Millipore Bioscience Research Reagents) and $0.5 \%$ Triton X-100 before use. Microscope fields with a magnification of $40 \times$ were documented either with an Axioplan2 (Zeiss), a FluoView 1000 confocal microscope (Olympus), or a LSM5 Pascal confocal microscope (Zeiss). Immunohistochemical detection of diphosphorylated Erk1/2 with the monoclonal anti-dpErk antibody (clone M-8159 Sigma-Aldrich) was performed as described by Corson et al. (2003).

RNA interference. For RNA interference (RNAi)-mediated knockdown of RPTP $\lambda$ or Wnt1 expression, the psiSTRIKE-U6 Hairpin Cloning System was used (Promega). The short hairpin RNA target sequences directed against nonoverlapping regions of the $C R P T P \lambda$ cytoplasmic domain were GTCAACATGACCAAAGCAA $\left(R P T P \lambda \_s i-a\right)$ and GCTTCAAGCAGGAGTATGA $\left(R P T P \lambda \_s i-b\right)$. The efficiency and specificity of the RNAi targeting constructs were assessed by luciferase reporter assay. Human embryonic kidney 293T (HEK293T) cells were cotransfected with the RNAi targeting constructs RPTP $\lambda \_s i-a, R P T P \lambda \_s i-b$, targeting constructs containing randomized sequences of $R P T P \lambda \_s i-a$ or $R P T P \lambda \_s i-b$, respectively (GAACTACGTAAACCAGACA or GCATAGACGAATCGGTATG), or an unrelated targeting construct (GAAGGTACACGAATTATGT) together with a psiCHECK-2 reporter construct, which contained the intracellular portion of $c R P T P \lambda$ fused to Renilla luciferase ( $1.5 \mu \mathrm{g}$ of each construct/six-well plate; Promega). After $48 \mathrm{~h}$, luciferase activity was measured using the Dual-Luciferase Reporter 1000 Assay System (Promega) in a GloMax96 plate Luminometer (Promega). For in vivo analysis, $R P T P \lambda \_s i-a$ or $R P T P \lambda \_s i-b$ was electroporated into the neural tube at a concentration of $1.5 \mu \mathrm{g} / \mu \mathrm{l}$ together with GFPpMIWIII $(0.8-0.5 \mu \mathrm{g} / \mu \mathrm{l})$ at the developmental ages indicated. Short hairpin RNA target sequences against Wnt1 were GGTCATCTACGGCAACAAA and GCGCCTCGAGGGTCATCTA. Simultaneous transfection of both RNA targeting constructs ( $1.5 \mu \mathrm{g} / \mathrm{six}$-well dish) repressed the activity of a psiCHECK-2 reporter construct, which contained the coding region of chick Wnt 1 fused to Renilla luciferase, to $34 \%$ compared with a targeting construct carrying the randomized sequence CGAATCAGTCAGTCCAGAA (data not shown).

Immunoprecipitation. RPTP $\lambda$-HA-pMES or RPTP $\lambda$ - $\triangle I C-H A$-pMES was electroporated into the midbrain at $\mathrm{HH} 10$. After $24 \mathrm{~h}$, the midbrain 


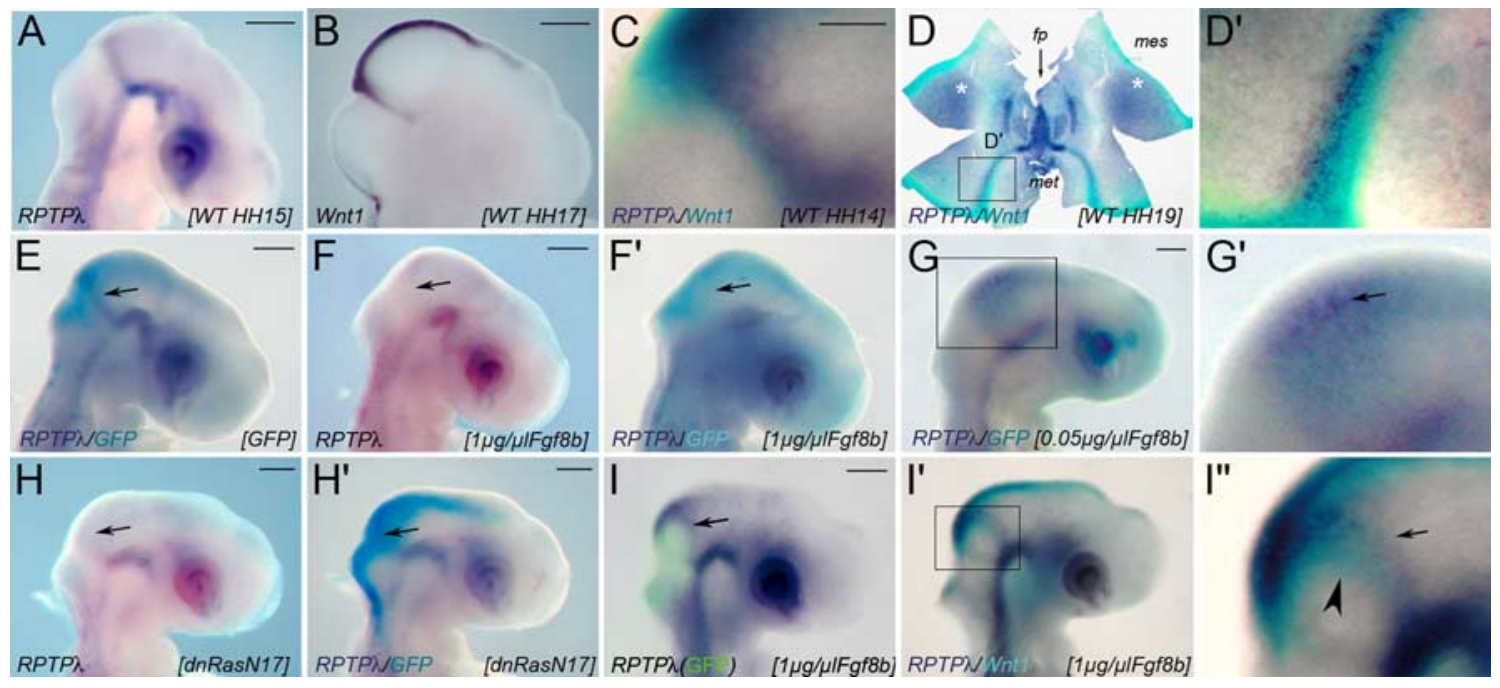

Figure 1. Expression and regulation of RPTP $\lambda$ at the MHB. A, Expression of RPTP $\lambda$ in a WT chick embryo at HH15. B, Expression of Wnt1 at HH17. C, Two-color in situ hybridization showing expression of RPTP $\lambda$ in dark blue and Wnt 1 in turquoise at HH14. The ring of Wnt1 expression at the MHB is bordered by RPTP $\lambda$-expressing cells at its rostral side. $\boldsymbol{D}, \boldsymbol{D}^{\prime}, 0$ pen book preparation of a HH19 (E3.5) chick midbrain showing RPTP $\lambda$ expression in dark blue and Wnt7 expression in turquoise. RPTP $\lambda$ and Wnt expression occurs in tightly associated, partially overlapping domains at the MHB; anterior is to the top, and posterior is to the bottom. $\boldsymbol{D}^{\prime}$, Higher magnification of the boxed area in D. E-I", Whole-mount in situ hybridizations on HH15-HH16 chick embry0s, electroporated with different expression constructs. In all panels, detected transcripts are indicated in the bottom left corner, and misexpressed transgenes are indicated in brackets in the bottom right corner. $\boldsymbol{E}$, Expression of RPTP $\lambda$ (dark blue) was not altered after misexpression of GFP (turquoise; $1.5 \mu \mathrm{g} / \mu \mathrm{l}$ ). $\boldsymbol{F}$, Transfection of $1 \mu \mathrm{g} / \mu$ l of the expression plasmid Fgf8b-pMIWIIII together with GFP-pMIWIII effectively repressed RPTP $\lambda$ expression at the MHB. $\boldsymbol{F}^{\prime}$, Two-color in situ hybridization on the embryo shown in $\boldsymbol{F}$ for RPTP $\lambda$ (dark blue) and GFP (turquoise). $\boldsymbol{G}, \boldsymbol{G}^{\prime}$, Transfection of $0.05 \mu \mathrm{g} / \mu$ l of Fgf8b-pMIWIII together with GFP-pMIWIII (turquoise) induced RPTP $\lambda$ expression (dark blue) in the dorsal midbrain. $\boldsymbol{G}^{\prime}$ is a higher magnification of the boxed area shown in $\boldsymbol{G}$. $\boldsymbol{H}, R P T P \lambda$ expression at the MHB was lost after misexpression of the dominant-negative Ras $N^{17}$ together with GFP. $\boldsymbol{H}^{\prime}$, Two-color in situ hybridization on the embryo shown in $\boldsymbol{H}$ (RPTP $\lambda$, dark blue; GFP, turquoise). $\boldsymbol{I}$, Forced expression of Fgf8b (together with GFP) in the MHB region shifted the ring of RPTP $\lambda$ expression rostrally around the transfected area. The image is an overlay of the GFP fluorescence photographed immediately after harvesting of the embryo and the RPTP $\lambda$ expression domain as detected subsequently by in situ hybridization (dark blue). $I^{\prime}, I^{\prime \prime}$, Spatial relationship of $R P T P \lambda$ (purple) and Wnt 7 (turquoise) expression in the embryo shown in $I . I^{\prime \prime}$ is a higher magnification of the boxed area in $I^{\prime}$. In all panels, upregulation of Wnt 7 is indicated by arrowheads, and expression of $R P T P \lambda$ (or lack thereof) is indicated by arrows. The asterisks in $\mathbf{D}$ mark upregulation of $R P T P \lambda$ expression in the mesencephalic alar plate after HH18. $C, D^{\prime}, D^{\prime}, I^{\prime}$, and $I^{\prime \prime}$ are false colored for better visual contrast. fp, Floor plate; mes, mesencephalon; met, metencephalon. Scale bars: $A, B, E-G, H-I, 500 \mu \mathrm{m} ; C, 250 \mu \mathrm{m}$.

was dissected in ice-cold PBS and lysed in $150 \mathrm{~mm} \mathrm{NaCl}, 10 \mathrm{~mm} \mathrm{Na}-$ Phosphate buffer, pH 7.2, $1 \%$ NP-40, $1 \%$ Desoxycholate, $0.1 \%$ SDS, 50 mм Na-F, 0.2 mм Na-orthovanadate, and protease inhibitors (Complete tablets; Roche Diagnostics). Precipitation of the transgenes was achieved using a monoclonal, agarose-coupled anti-HA antibody (SigmaAldrich). Immunoprecipitation and Western blot analysis were performed as described previously (Mühleisen et al., 2006). Primary antibodies used for Western blot analysis were rat anti-HA high-affinity antibody (1:1000; Roche Diagnostics) or polyclonal anti- $\beta$-catenin (1: 1000; Upstate Biotechnology). For stripping off the first set of antibodies, the blots were incubated in $0.1 \mathrm{M}$ glycine, $\mathrm{pH} 2.5$, for $30 \mathrm{~min}$ at $37^{\circ} \mathrm{C}$.

Analysis of cell proliferation and programmed cell death. RPTP $\lambda-H A-$ pMES or pMES were electroporated into the midbrain of HH9 chick embryos. After $24 \mathrm{~h}$, bromodeoxyuridine (BrdU) was injected into the midbrain vesicle; labeling duration was $2 \mathrm{~h}$. Detection of BrdU-positive cells was performed on 15- $\mu$ m-thick cryosections using the BrdU Labeling and Detection Kit I (Roche Diagnostics) and colabeling with a GFPspecific antibody to visualize transfected cells. For analysis of programmed cell death, $R P T P \lambda$-pMIWIII together with $n R F P$-pCAGGS, or $n R F P$-pCAGGS alone, was electroporated into the midbrain vesicle. Detection of apoptotic cells was performed on cryosections using the Fluorescein In Situ Cell Death Detection Kit (Roche Diagnostics).

\section{Results}

$R P T P \lambda$ is expressed in a domain tightly associated with the ring of Wnt 1 expression at the $\mathrm{MHB}$ and is regulated by $\mathrm{Fg} 8$ We reported previously that between the 19 and 40 somite stages (HH13-HH19), RPTP $\lambda$ is expressed in a tight, transverse ring anterior to the isthmic constriction and to the transverse domain of Wnt1 expression (Fig. 1A,B) (Badde et al., 2005). To correlate the precise location of this ring to that of Wnt1 at the organizer, $R P T P \lambda$ - and Wnt1-specific transcripts were visualized together in the same specimen by two-color in situ hybridization. Expression of the two molecules was found in tightly associated domains at HH14 (Fig. 1C) and HH19 (Fig. 1D, D'). Although both expression domains appear to slightly overlap, in all specimens analyzed, $R P T P \lambda$-specific transcripts were found rostral to the ring of $W n t 1$ expression that abuts the MHB. Therefore, during this developmental period, the Wnt1 domain at the MHB is bordered by a ring of $R P T P \lambda$-expressing cells at its anterior side. Based on these expression data, the RPTP $\lambda$ expression domain at the MHB may lie within the future preisthmic domain, the caudal-most aspect of the mesencephalic alar plate, which is characterized by coexpression of Otx2, Pax2, and possibly Wnt1 (Hidalgo-Sánchez et al., 2005).

As a first step to understanding RPTP $\lambda$ function at the MHB, we investigated whether its expression was regulated by the secreted protein Fgf8. Fgf8 is expressed in rhombomere 1 adjacent to the isthmic organizer and can induce organizer characteristic gene expression and cell fate changes when ectopically applied in the caudal diencephalon or mid-hindbrain region (Crossley et al., 1996; Martinez et al., 1999). Transfection of $1 \mu \mathrm{g} / \mu \mathrm{l}$ to $0.1 \mu \mathrm{g} / \mu \mathrm{l}$ of an expression plasmid carrying the splicing isoform $F g f 8 b$ into the mid-hindbrain region of chick embryos has been shown previously to induce ectopic cerebellar development, whereas transfection of $0.01 \mu \mathrm{g} / \mu \mathrm{l}$ promoted tectal growth (Sato et al., 2001). We introduced an Fgf $8 b$ expression plasmid at different concentrations into the mid-hindbrain region together with an expression plasmid carrying GFP for visualization (Fig. $1 F-G^{\prime}$ ). Expression of Wnt1 and RPTP $\lambda$ at the MHB were unaltered after transfection with the GFP expressing plasmid alone compared 
with wild-type (WT) control embryos, demonstrating that in ovo electroporation per se does not affect gene expression at the MHB (Fig. $1 E$ ) (data not shown) ( $n=36$ of 37 for $R P T P \lambda ; n=12$ of 12 for Wnt1). Consistent with our observation that RPTP $\lambda$ is not expressed in the hindbrain region during normal development, we found that widespread electroporation across the midhindbrain territory of $1 \mu \mathrm{g} / \mu \mathrm{l}$ of the Fgf $8 b$-pMIWIII expression plasmid, a concentration known to stimulate cerebellar development, effectively repressed RPTP $\lambda$ expression at the MHB $(n=9$ of 10) (Fig. $1 F, F^{\prime}$ ). Electroporation of $0.01 \mu \mathrm{g} / \mu \mathrm{l}$ of the $F g f 8 b$ expression plasmid, a concentration that enhanced midbrain growth in a previous study (Sato et al., 2001), did not alter $R P T P \lambda$ expression ( $n=7$ of 7) (data not shown). However, RPTP $\lambda$ specific transcripts were upregulated in the midbrain vesicle at an

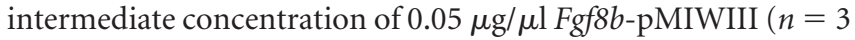
of 5) (Fig. $1 G, G^{\prime}$ ). Fgf $8 b$ misexpression at either concentration was unable to stimulate $R P T P \lambda$ expression in the hindbrain, supporting the idea that metencephalic tissue is not permissive for $R P T P \lambda$ expression ( $n=18$ of 18 ) (Fig. $1 F-G^{\prime}$ ) (data not shown). Together, these results suggest that $R P T P \lambda$ expression at the isthmic organizer requires a distinct Fgf8 signaling level, which is below the level required for metencephalic development but above the level that stimulates tectal growth. Fgf8 signal transduction at the MHB appears to be mediated primarily through the Ras-mitogen-activated protein (MAP) kinase pathway (Corson et al., 2003; Sato and Nakamura, 2004). To confirm the dependency of RPTP $\lambda$ expression on Fgf8 signaling at the MHB, we misexpressed a dominant-negative form of $\operatorname{Ras}\left(d n R a s^{N 17}\right)$, which was shown previously to effectively block Fgf8 signal transduction at the isthmic organizer (Sato and Nakamura, 2004). $R P T P \lambda$ expression was consistently lost in cells forced to express $d n R^{2}{ }^{N 17}$, demonstrating that RPTP $\lambda$ expression at the MHB indeed requires $F g f 8 /$ Ras-MAP kinase pathway activity (Fig. $\left.1 H, H^{\prime}\right)(n=8$ of 9$)$.

Next, we targeted the Fgf8b expression plasmid (at a concentration of $1 \mu \mathrm{g} / \mu \mathrm{l}$ ) to a narrow region surrounding the isthmic organizer and analyzed the expression of RPTP $\lambda$ and Wnt1 together in the same specimen $24 \mathrm{~h}$ later (Fig. $\left.1 I-I^{\prime \prime}\right)$. The domain of $R P T P \lambda$ expression was shifted rostrally around the region targeted for F $8 f 8$ misexpression (recognizable by the fluorescence of the coelectroporated GFP; $n=9$ of 10) (Fig. 1 I, arrow), consistent with the idea that RPTP $\lambda$ expression is inhibited by strong $F g f 8 b$ signals but induced at a distance from the $F g f 8 b$ source, where Fgf8b protein levels are expected to be lower. When Wnt1and $R P T P \lambda$-specific transcripts were visualized together, both expression domains were arranged in concentric circles, with the $R P T P \lambda$ domain (arrow) always located anterior to the Wnt1 domain (Fig. $1 I^{\prime}, I^{\prime \prime}$, arrowhead) ( $n=6$ of 6). Fgf8-induced upregulation of $W n t 1$ and $R P T P \lambda$ thus mimics their normal spatial relationship at the MHB, where the Wnt1 domain separates the Fgf8 and RPTP $\lambda$ expression domains (Badde et al., 2005) (Fig. 2G). This raises the possibility that the threshold of Fgf8 signaling differs from the induction of Wnt1 and RPTP $\lambda$ expression at the $\mathrm{MHB}$, and that Wnt1 expression requires higher levels of Fgf8 signaling than expression of RPTP $\lambda$. To investigate this in more detail, we narrowed down the concentrations of $F g f 8$ necessary for inducing Wht1 and RPTP $\lambda$ expression, respectively, by implanting fragments of beads soaked in different concentrations of Fgf8b. As reported previously, Wnt1 expression was induced around a bead fragment soaked in $0.15 \mathrm{mg} / \mathrm{ml} \mathrm{Fgf8b}$ (Fig. $2 A, A^{\prime}$, arrowheads) ( $n=12$ of 12) (Crossley et al., 1996). Induction of $R P T P \lambda$ expression was also consistently observed, yet its expression domain was located at a distance to the Fgf8b soaked bead

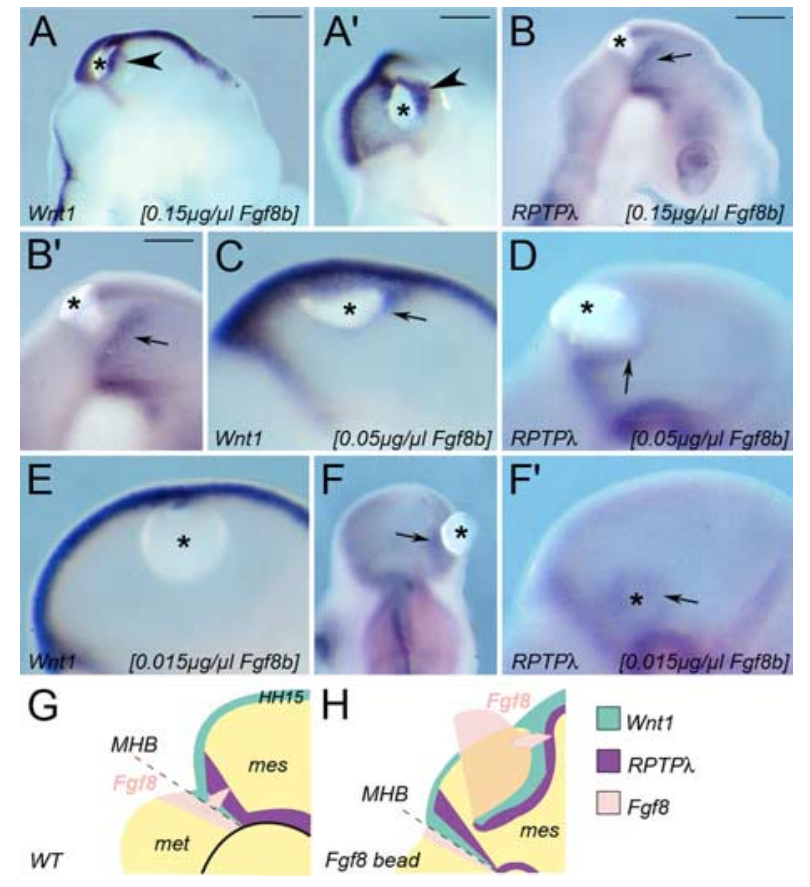

Figure 2. Fgf8-induced upregulation of Wnt1 and RPTP $\lambda$ mimics their normal spatial relationship at the MHB. $\boldsymbol{A}^{\prime}-\boldsymbol{F}^{\prime}$, Expression of Wnt $1\left(\boldsymbol{A}, \boldsymbol{A}^{\prime}, \boldsymbol{C}, \boldsymbol{E}\right)$ and $R P T P \lambda\left(\boldsymbol{B}, \boldsymbol{B}^{\prime}, \boldsymbol{D}, \boldsymbol{F}, \boldsymbol{F}^{\prime}\right)$ after implantation of beads soaked in different concentrations of recombinant Fgf8b. Wnt1 expression $\left(A, A^{\prime}\right)$ is ectopically upregulated in the mesencephalon closely around a partial bead soaked in $0.15 \mathrm{mg} / \mathrm{ml} \mathrm{Fgf8b}, R P T P \lambda$ expression only at a distance to the bead $\left(\boldsymbol{B}, \boldsymbol{B}^{\prime}\right) . \boldsymbol{A}^{\prime}$ is a back view of the embryo shown in $\boldsymbol{A}$. $\boldsymbol{C}, \boldsymbol{D}$, Ectopic expression surrounding beads soaked in 0.05 $\mathrm{mg} / \mathrm{ml}$ Fgf8b. $\boldsymbol{E}, \boldsymbol{F}, \boldsymbol{F}^{\prime}$, Expression surrounding beads soaked in $0.015 \mathrm{mg} / \mathrm{ml} \mathrm{Fgf8b.} \mathrm{In} \boldsymbol{F}^{\prime}$, the Fgf8b-releasing bead was removed for better visual clarity. $G$, Schematic drawing of the gene expression patterns of $F g f 8, W n t 1$, and RPTP $\lambda$ at the MHB of a normal chick embryo (HH15). $\boldsymbol{H}$, Schematic drawing of Wnt 1 and RPTP $\lambda$ expression around a Fgf8-releasing bead fragment (pink) implanted into the mesencephalic alar plate, which acts as ectopic Fgf8 source in the dorsal midbrain. The asterisks in all panels indicate the location of Fgf8b-releasing beads. Scale bar, $100 \mu \mathrm{m}$.

and must therefore lie distal of the ring of Wnt1 expression, which generally surrounded the Fgf8 source (Fig. $2 B, B^{\prime}$, arrows) $(n=$ 15 of 15). When the Fgf8b concentration was lowered to 0.05 $\mathrm{mg} / \mathrm{ml}$, some ectopic Wnt 1 expression could still be observed surrounding the Fgf8 soaked bead, and the ring of RPTP $\lambda$ specific transcripts was located closer to the bead (Fig. 2C,D) $(n=$ 5 of 8 for $W n t 1 ; n=7$ of 7 for RPTP $\lambda$ ). Fgf8b at $0.015 \mathrm{mg} / \mathrm{ml}$ was not sufficient to induce Wnt1 expression (Fig. $2 E)(n=1$ of 3$)$ but could still elicit robust ectopic RPTP $\lambda$ expression (Fig. $2 F, F^{\prime}$ ) $(n=4$ of 4$)$. Although additional work is clearly needed to fully understand $F g$ receptor signaling dynamics at the MHB, these results strongly suggest that transcriptional activation of Wnt1 and $R P T P \lambda$ requires different levels of $F g f 8$ signaling.

\section{Overexpression of RPTP $\lambda$ suppresses Wnt1 expression at the MHB}

To examine the function of $R P T P \lambda$ at the MHB, we misexpressed the murine homolog of $R P T P \lambda$ in the area surrounding the isthmic constriction (Fig. $3 A$ ). By fusing the coding region of $R P T P \lambda$ to a triple HA-tag, the ectopically expressed $R P T P \lambda$ protein could be distinguished from its endogenous form by the use of an HAspecific antibody. As expected for a transmembrane protein, $R P T P \lambda-H A$ staining was concentrated to cellular plasma membranes, suggesting that the recombinant protein was processed normally (Fig. 3B). When GFP alone was ectopically expressed in the MHB region, Wnt1 expression was unaltered (Fig. $3 C)(n=$ 
A
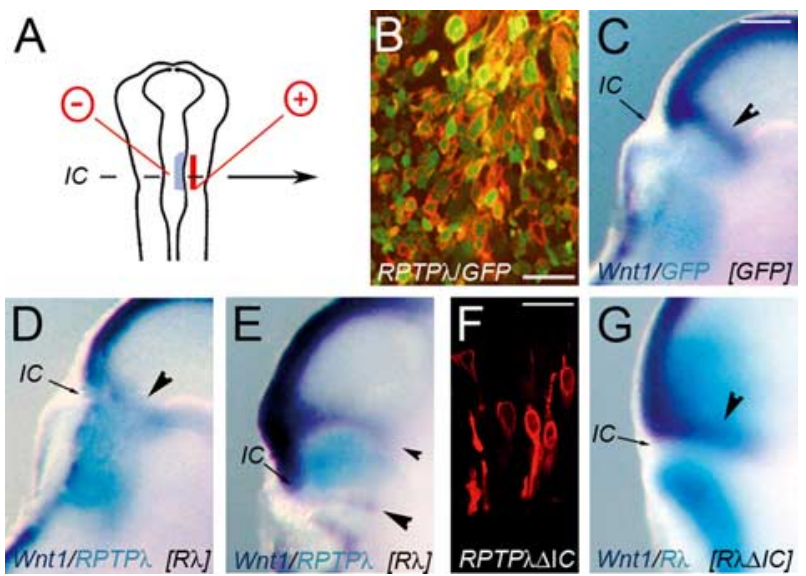

Figure 3. Altered Wnt1 expression after RPTP $\lambda$ misexpression at the MHB. $A$, Schematic representation of the experimental protocol. $B$, Double immunohistochemical labeling using antibodies against GFP (green) and the HA-epitope (red) on a frozen section through the MHB region of an embryo electroporated with RPTP $\lambda$-HA-pMIWIII and GFP-pMIWIII. HA-specific staining is concentrated to cellular plasma membranes. C, Expression of Wnt1 after misexpression of GFP. D, E, Different example of Wnt1 expression after misexpression of RPTP $\lambda$-HA together with GFP. $F$, Immunohistochemical analysis of $R P T P \lambda-\Delta / C-H A$ expression as detected by expression of the HA-fusion epitope. Expression is concentrated to cellular plasma membranes. G, Wnt1 expression after transfection with RPTP $\lambda-\Delta / C-H A$. In $\mathbf{C}-\boldsymbol{E}$ and $\mathbf{G}$, Wnt1 expression is shown in dark blue, and GFP is shown in turquoise. The arrowheads in $\boldsymbol{C} \boldsymbol{E}$ and $\boldsymbol{G}$ mark the transverse Wnt1 domain at the MHB. Scale bars: $\boldsymbol{B}, \boldsymbol{F}, 20 \mu \mathrm{m}$; (in $\boldsymbol{C}) \boldsymbol{C}, \boldsymbol{D}, \boldsymbol{E}, \boldsymbol{G}, 100 \mu \mathrm{m}$. IC, Isthmic constriction.

15 of 16). In contrast, after ectopic expression of $R P T P \lambda-H A$, the normal Wnt1 expression pattern was markedly disturbed (Fig. $3 D, E)$ ( $n=17$ of 20 ). In embryos in which $R P T P \lambda-H A$ was misexpressed in a broad region surrounding the isthmic constriction, Wnt1 expression was reduced or lost from the electroporated side of the embryo, suggesting that strong $R P T P \lambda$ expression inhibits $W n t 1$ expression at the MHB (Fig. 3D, arrowhead) $(n=12$ of 20$)$. In some embryos, particularly in those where $R P T P \lambda$-HA expression was directly targeted to the organizer (and just to the region where $W n t 1$ would normally be expressed), a rostral displacement or split of the Wnt1 expression domain around the RPTP $\lambda-H A$ expressing cells was observed (Fig. $3 E$, arrowheads) ( $n=5$ of 20 ). Identical results were obtained when an untagged form of the protein was misexpressed, indicating that the presence of the triple-HA tag was not critical for RPTP $\lambda$ function (data not shown). To test for the possible contribution of the cytoplasmic part of $R P T P \lambda$, the domain most likely to be essential for its phosphatase activity and the interaction with components of intracellular signal transduction pathways, we constructed truncated forms of $R P T P \lambda$ in which a triple HA-tag replaced the juxtamembrane and phosphatase domains $(R P T P \lambda$ $\triangle I C-H A)$. When overexpressed in the mesencephalic vesicle, $R P T P \lambda-\triangle I C-H A$ staining was concentrated to cellular plasma membranes, indicating correct targeting of the truncated protein (Fig. $3 F$ ). In contrast to the full-length form of $R P T P \lambda$, electroporation of $R P T P \lambda-\Delta I C-H A$ did not perturb Wnt1 expression (Fig. $3 G)(n=21$ of 22$)$, indicating that the cytoplasmic domain was required for RPTP $\lambda$ function at the MHB.

To further characterize the potential role of $R P T P \lambda$ in modulating gene expression at the MHB, we analyzed the expression of other MHB marker genes after electroporation of RPTP $\lambda-H A$. The expression domain of $F g f 8$ was normal $24 \mathrm{~h}$ after misexpression of RPTP $\lambda$ ( $n=6$ of 6$)$ (supplemental Fig. $1 A, B$, available at www.jneurosci.org as supplemental material). The altered $W n t 1$ expression that we observed $24 \mathrm{~h}$ after $R P T P \lambda$ transfection can therefore not be a secondary effect of an altered $F g f 8$ expression profile at the MHB. In addition, forced expression of RPTP $\lambda$ did not affect expression of $\operatorname{Lmx} 1 b(n=16$ of 17$), E n 1$ ( $n=8$ of 8$)$, $\operatorname{Pax2}(n=9$ of 11$), \operatorname{Pax} 5$ ( $n=9$ of 10), Otx2 ( $n=4$ of 4$), \operatorname{Sef1}(n=$ 7 of 8$)$, sprouty2 ( $n=7$ of 7$)$, or Grg4 ( $n=5$ of 5$)$ within 24 h after transfection (supplemental Fig. $1 C-K$, available at www. jneurosci.org as supplemental material) (data not shown).

\section{$R P T P \lambda$ interferes with $F g f 8$-mediated $W n t 1$ induction}

Next, we took advantage of the fact that $F g f 8$ can mimic the organizer's activity when ectopically expressed in adjacent brain regions and performed two consecutive rounds of in ovo electroporation within the mesencephalic alar plate (Fig. $4 A, A^{\prime}$ ). Electroporation of the Fgf $8 b$ expression plasmid into an embryo without previous manipulation reproducibly led to ectopic Wnt1 expression (Fig. $\left.4 B, B^{\prime}\right)(n=5$ of 5). Mock-transfection with GFP or $d s R e d$ did not alter $W n t 1$ expression (Fig. 4G). Overexpression of $F g f 8 b$ in embryos already experiencing $6 \mathrm{~h}$ of GFP or RPTP $\lambda$ $\triangle I C$ expression also resulted in robust upregulation of $W n t 1$ expression (Fig. $4 C, D$ ) ( $n=5$ of 5 for GFP; $n=7$ of 8 for $R P T P \lambda$ $\triangle I C)$. In contrast, when full-length $R P T P \lambda$ was misexpressed in the mesencephalic vesicle (Fig. $4 E$ ), only $22 \%$ of the transfected embryos exhibited robust upregulation of Wnt1 after Fgf $8 b$ electroporation (Fig. $\left.4 F, F^{\prime}\right)(n=4$ of 18$)$. Because overexpression of $R P T P \lambda$ at the MHB had specifically perturbed the expression of Wnt1 but not that of MHB marker genes like En1, Pax2, or Pax5 (supplemental Fig. 1, available at www.jneurosci.org as supplemental material), we analyzed expression of these molecules after sequential $R P T P \lambda / F g f 8 b$ electroporation. In agreement with our previous results, expression of all three molecules was strongly induced by $F g f 8$, regardless of previous overexpression of $R P T P \lambda$ (supplemental Fig. $1 L-O$, available at www.jneurosci.org as supplemental material) ( $n=6$ of 7 for $E n 1 ; n=5$ of 5 for $P a x 2 ; n=$ 5 of 5 for $P a x 5$ ). Together, these results suggest that strong RPTP $\lambda$ expression specifically interferes with the ability of cells in the embryonic mesencephalon to initiate Wnt1 expression in response to $F g f 8$ signals. Considering that, during normal development (or in response to local Fgf8 application), RPTP $\lambda$ expression always extended over the Wnt 1 domain at its rostral side, our data suggest that RPTP $\lambda$ may function to rostrally restrict the domain of Wnt1 expression at the MHB.

If $R P T P \lambda$ expression at the MHB indeed serves to restrict $W n t 1$ expression, one would expect that reducing $R P T P \lambda$ activity at the MHB should allow Wnt1 expression to expand anteriorly. To test this idea, two RNAi constructs targeted to nonoverlapping sequences in the intracellular domain of $R P T P \lambda$ were designed. The activity and specificity of the constructs were confirmed by luciferase reporter assays compared with the effects of an RNAi construct targeted to an unrelated protein or with that of RNAi constructs containing randomized targeting sequences (Fig. 5A). Repression by unrelated or randomized sequences was negligible (Fig. 5A). Electroporation of either RPTP $\lambda \_s i-a(n=8$ of 9) (Fig. 5D) or RPTP $\lambda \_s i-b(n=6$ of 9) (data not shown) but not of the randomized control targeting construct random_a $(n=5$ of 5 ) (Fig. $5 C$ ) into the MHB region at HH10 markedly reduced the endogenous expression of $R P T P \lambda$. Knock-down of $R P T P \lambda$ also led to an anterior expansion of $W n t 1$-positive cells into the domain where $R P T P \lambda$ is normally expressed (Fig. $5 G-$ $\left.G^{\prime \prime}, I, J\right)(n=9$ of 14$)$. This effect was more pronounced near the dorsal midline than in the ventral midbrain, presumably because the domain of RPTP $\lambda$ expression is broader and any effect of knock-down of its expression may therefore be more profound in the dorsal midbrain (Fig. 5E,K). Wnt1 expression at the MHB, 


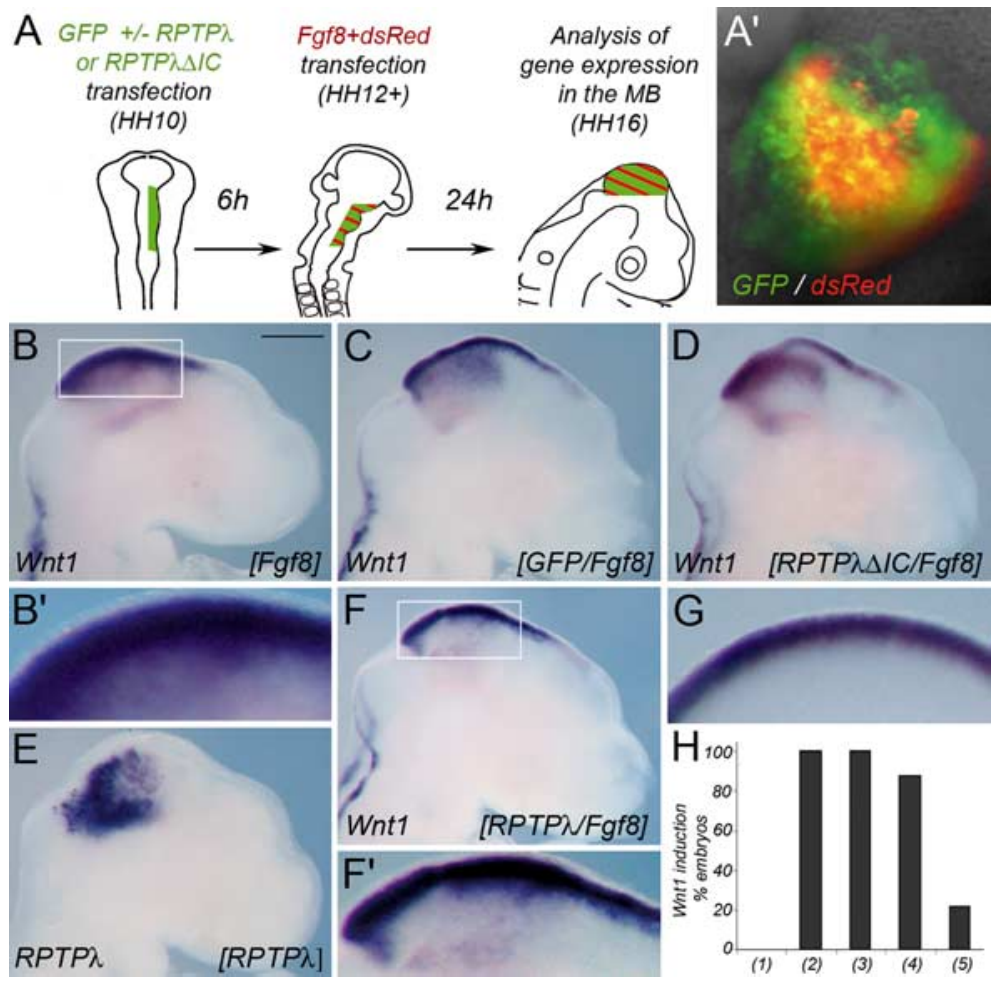

Figure 4. Misexpression of RPTP $\lambda$ interferes with transcriptional Wnt1 activation after ectopic Fgf8 expression. $\boldsymbol{A}$, Schematic drawing of the experimental procedure. At HH10, RPTP $\lambda$ plus GFP, RPTP $\lambda-\Delta / C$ plus GFP, or GFP alone were introduced into the right side of the midbrain vesicle. After $6 \mathrm{~h}$ (at $\mathrm{HH} 12+$ ), two expression plasmids carrying Fgf8 and $d s$ Red, respectively, were electroporated into the same side of the midbrain vesicle. The embryos were allowed to develop for an additional $24 \mathrm{~h}$. $\boldsymbol{A}^{\prime}$, Example of an embryo chosen for additional analysis, based on the massive coexpression of the red and green fluorescent proteins in the midbrain vesicle. $\boldsymbol{B}, \boldsymbol{B}^{\prime}$, Ectopic expression of Fgf8 in the midbrain without previous manipulation leads to widespread upregulation of Wnt1. C, D, Fgf8 induced Wnt1 expression is not affected by previous introduction of GFP (C) or $R P T P \lambda-\Delta / C(\boldsymbol{D}) . \boldsymbol{E}$, Example of an embryo electroporated with RPTP $\lambda$-pMES indicating mosaic expression of the transgene. $\boldsymbol{F}, \boldsymbol{F}^{\prime}$, Transcriptional upregulation of Wnt1 is mostly prevented after ectopic expression of $R P T P \lambda$ $6 \mathrm{~h}$ before Fgf8 transgene introduction. $\boldsymbol{B}^{\prime}, \boldsymbol{F}^{\prime}$, Higher magnification of the boxed areas shown in $\boldsymbol{B}$ and $\boldsymbol{F}$. $\boldsymbol{G}$, Expression of Wnt1 in an embryo electroporated with GFP followed by misexpression of $d s$ Red $6 \mathrm{~h}$ later demonstrating normal expression in the mesencephalic roof plate under these experimental conditions. $\boldsymbol{H}$, Quantification of the results: (1), Percentage of embryos exhibiting strong ectopic expression of Wnt1 $24 \mathrm{~h}$ after transfection with GFP or dsRed alone; (2), percentage of embryos exhibiting strong ectopic expression of Wnt1 $24 \mathrm{~h}$ after transfection with Fgf8b (together with dsRed) without previous manipulation; (3), percentage of embryos exhibiting strong ectopic expression of Wnt1 $24 \mathrm{~h}$ after transfection with GFP followed by Fgf8b $(+d s R e d) ;(4)$, percentage of embryos exhibiting strong ectopic expression of Wnt $124 \mathrm{~h}$ after transfection with $R P T P \lambda-\Delta / C-H A(+G F P)$ followed by Fgf8b (+dsRed); (5), percentage of embryos exhibiting strong ectopic expression of Wnt1 $24 \mathrm{~h}$ after transfection with RPTP $\lambda-H A(+G F P)$ followed by Fgf8b $(+d s R e d)$. Scale bar, $500 \mu \mathrm{m}$.

however, was not only expanded rostrally, but expression was also scattered within the rostral half of its normal domain in some embryos (Fig. 5F-J). We do not know the molecular basis for this effect at present. A possible explanation may come from the fact that RPTP $\lambda$ and Wnt1 expression at the MHB appear to slightly overlap during normal development (Figs. $1 C-D^{\prime}, 5 E$ ). It is therefore possible that $R P T P \lambda$ stabilizes the $W n t 1$ expression border in the small region where both proteins are normally coexpressed. In addition, because the extracellular part of $R P T P \lambda$ contains multiple cell adhesion-like motives, reduced $R P T P \lambda$ expression at the MHB may alter cell adhesive properties critical for the maintenance of the coherent cellular organization within its normal expression domain. It is worth pointing out that disorganization of the Wnt1 expression domain was also observed after targeted deletion of Fgf receptor 1 from the MHB territory (Trokovic et al., 2003).
At least three signaling pathways, the Ras-MAP kinase, phosphatidyl inositol 3 kinase, and phospholipase $\mathrm{C} \gamma$ pathway, can be activated after Fgf receptor stimulation, and of the three, the Ras-MAP kinase pathway has been demonstrated to be important for midhindbrain development (Corson et al., 2003; Sato and Nakamura, 2004; Dailey et al., 2005). Because signal transduction in each of these pathways requires protein phosphorylation on tyrosine residues, it is possible that $R P T P \lambda$, because of its tyrosine phosphatase activity, interrupts signal transduction through dephosphorylation of intermediate signaling components. To test whether RPTP $\lambda$ impinges on Ras-MAP kinase signaling, we visualized phosphorylation of the pathway intermediate Erk1/2 with the phosphorylation-specific antibody dpErk1/2 (Corson et al., 2003). Six hours after transfection of $2 \mu \mathrm{g} / \mu \mathrm{l} \operatorname{RPTP} \lambda$-pMES but not after transfection of pMES alone, dpErk1/2 labeling intensity was slightly reduced in the right electroporated side of the electroporated embryos (Fig. $5 N-P)$ ( $n=6$ of 9 ). Repression of $W n t 1$ expression by $R P T P \lambda$ could also be detected 6 and $8 \mathrm{~h}$ after $R P T P \lambda$ transfection (Fig. $5 Q-T)(n=10$ of 18$)$. In contrast to Wnt1 but in agreement with our previous results (supplemental Fig. 1, available at www.jneurosci.org as supplemental material), we did not observe any major disturbance of En1 expression 6 or $8 \mathrm{~h}$ after RPTP $\lambda$ transfection (Fig. $5 U, V)$ (data not shown) ( $n=11$ of 11$)$. These findings indicate that the influence of $R P T P \lambda$ on Wnt1 expression at the MHB may, at least in part, result from modulation of Ras-MAP kinase signaling intensity by $R P T P \lambda$.

\section{RPTP $\lambda$ interacts with $\beta$-catenin in the embryonic midbrain and inhibits the activity of a $\beta$-catenin responsive promoter}

$R P T P \lambda$ expression in the developing midbrain is highly dynamic during the first $4 \mathrm{~d}$ of chick development (Figs. 1, 6A-C) (Badde et al., 2005). Before HH12 (16 somites), RPTP $\lambda$ is expressed throughout the mesencephalic vesicle (Fig. 6A) (Badde et al., 2005). Between HH12 and HH19, RPTP $\lambda$ expression is lost from the midbrain alar plate but is maintained in a tight transverse domain rostral to the Wnt1 domain (Figs. $1 A, C$, 6B) (Badde et al., 2005). Thereafter, RPTP $\lambda$ expression is induced once again in the mesencephalic vesicle and covers the entire mesencephalic alar plate from late E4 onwards, without crossing into the isthmic organizer region (Figs. 1D, 6C, arrowhead) (Badde et al., 2005). Because of this prominent "on-off" expression in the midbrain alar plate, we speculated that $R P T P \lambda$ may contribute to midbrain development by mechanisms in addition to restricting $W n t 1$ expression at the MHB organizer.

The juxtamembrane domain of $R P T P \lambda$ exhibits a high degree of homology to the cytoplasmic domain of E-cadherin (Wang et al., 1996). Moreover, the human homolog of RPTP $\lambda$, PCP2, colocalizes with $\beta$-catenin at sites of cell-to-cell contact in human adenocarcinoma cells and coprecipitates with $\beta$-catenin in PC-12 cells overexpressing both proteins (Yan et al., 2002). The interaction of both proteins occurs independently of the phosphorylation state of $\beta$-catenin, but $\beta$-catenin can be dephosphorylated on tyrosine residues by PCP2 (Yan et al., 2002). We therefore investigated whether $\beta$-catenin and RPTP $\lambda$ may physically inter- 
act in the developing mesencephalon. We overexpressed either $R P T P \lambda-H A$ or $R P T P \lambda-\triangle I C-H A$ in the mesencephalic vesicle for $24 \mathrm{~h}$ and subsequently immunoprecipitated RPTP $\lambda$ bound proteins using an antibody directed against the HA-epitope (Fig. 6D,E). Overexpressed RPTP $\lambda$-HA was detected as a discrete band of $\sim 185$ $\mathrm{kDa}$ recognized by the HA-specific antibody (Fig. 6D, top panel, lysate). After stripping and reprobing of the blot, endogenous $\beta$-catenin expression could also be readily detected (Fig. $6 D$, bottom panel, lysate). Precipitation with an unspecific antibody or with agarose-coupled protein-G did not deplete RPTP $\lambda$-HA or $\beta$-catenin from the extracts (Fig. 6, preclear). After immunoprecipitation with the HA-specific antibody, the $185 \mathrm{kDa}$ band was cleared from the supernatant (Fig. $6 D$, top panel, sup) and enriched in the immunoprecipitate (Fig. $6 D$, top panel, IP) $(n=5$ of 5$)$. $\beta$-Catenin was also present in the immunoprecipitates (Fig. $6 D$, bottom panel, IP) $(n=5$ of 5$)$. Neither $\beta$-catenin nor RPTP $\lambda$ could be precipitated with an antibody not directed against HA or $\beta$-catenin (data not shown). When the truncated allele RPTP $\lambda$ $\Delta \mathrm{IC}-\mathrm{HA}$, which lacks the cytoplasmic part of the protein and thus the putative interaction domain with $\beta$-catenin, was electroporated into the mesencephalic vesicle, a specific band of $105 \mathrm{kDa}$ was detected (Fig. $6 E$, top panel, lysate). After immunoprecipitation with the HA-specific antibody, RPTP $\lambda-\Delta$ IC-HA could be successfully immunoprecipitated but failed to enrich $\beta$-catenin (Fig. $6 E$, IP) $(n=5$ of 5$)$. These data demonstrate that RPTP $\lambda$ can coprecipitate $\beta$-catenin from mesencephalic extracts via its intracellular domain.

We next investigated whether the observed interaction of $\beta$-catenin with $\operatorname{RPTP} \lambda$ was biologically relevant for canonical Wnt signaling. $\beta$-Catenin plays an essential role in the structural organization of cells by linking the cytoplasmic domain of type I cadherins, such as E-cadherin, to the actin cytoskeleton and, in addition, functions as a key component of the canonical Wnt signaling pathway. Loss of E-cadherinmediated cell adhesion increased canonical Wnt signaling, presumably because, in the absence of E-cadherin, more $\beta$-catenin is available for Wnt signal transduction (Orsulic et al., 1999; Stockinger et al., 2001). We therefore wondered whether overexpression of RPTP $\lambda$ in the mesencephalon may sequester a portion of $\beta$-catenin at the plasma membrane, making it unavailable for Wnt signaling. To test this idea, we examined the activity of the $\beta$-catenin responsive reporter TOP:dgfp in the midbrain, alone or in the presence of ectopically expressed $R P T P \lambda$. TOP:dgfp contains four consensus Lef-binding sites and a minimal c-Fos promoter-driving expression of a destabilized form of GFP (dGFP) (Dorsky et al., 2002). Transgenic zebrafish that stably express dGFP under control of this promoter exhibit intense GFP expression throughout the entire midbrain alar plate, demon- strating that the canonical $W n t / \beta$-catenin pathway is active in this region of the neural tube (Dorsky et al., 2002). When the TOP: dgfp reporter was introduced into the MHB region of $\mathrm{HH} 11$ chick embryos by in ovo electroporation, strong GFP expression in the midbrain alar plate could be observed $24 \mathrm{~h}$ later (at HH1516), demonstrating that the midbrain alar plate is a domain of active $W n t / \beta$-catenin signaling in the chick at this developmental age (Fig. $6 F-H, N)(n=8$ of 8$)$. Therefore, at HH15-HH16, neither RPTP $\lambda$ nor $W n t 1$ are expressed in the mesencephalic alar plate, but the $W n t / \beta$-catenin pathway is active. We therefore reasoned that misexpression of RPTP $\lambda$ in the midbrain alar plate at HH15-H16 should allow us to analyze a possible influence of $R P T P \lambda$ on canonical Wnt signal transduction that is independent of its ability to influence Wnt1 expression at the MHB. When $R P T P \lambda$ was coelectroporated with TOP:dgfp, GFP fluorescence intensity was reduced severely (Fig. $6 I-K, N)(n=10$ of 11$)$. Transfection of $R P T P \lambda-\Delta I C$, which lacks the intracellular domain critical for coprecipitation of $\beta$-catenin, did not suppress 


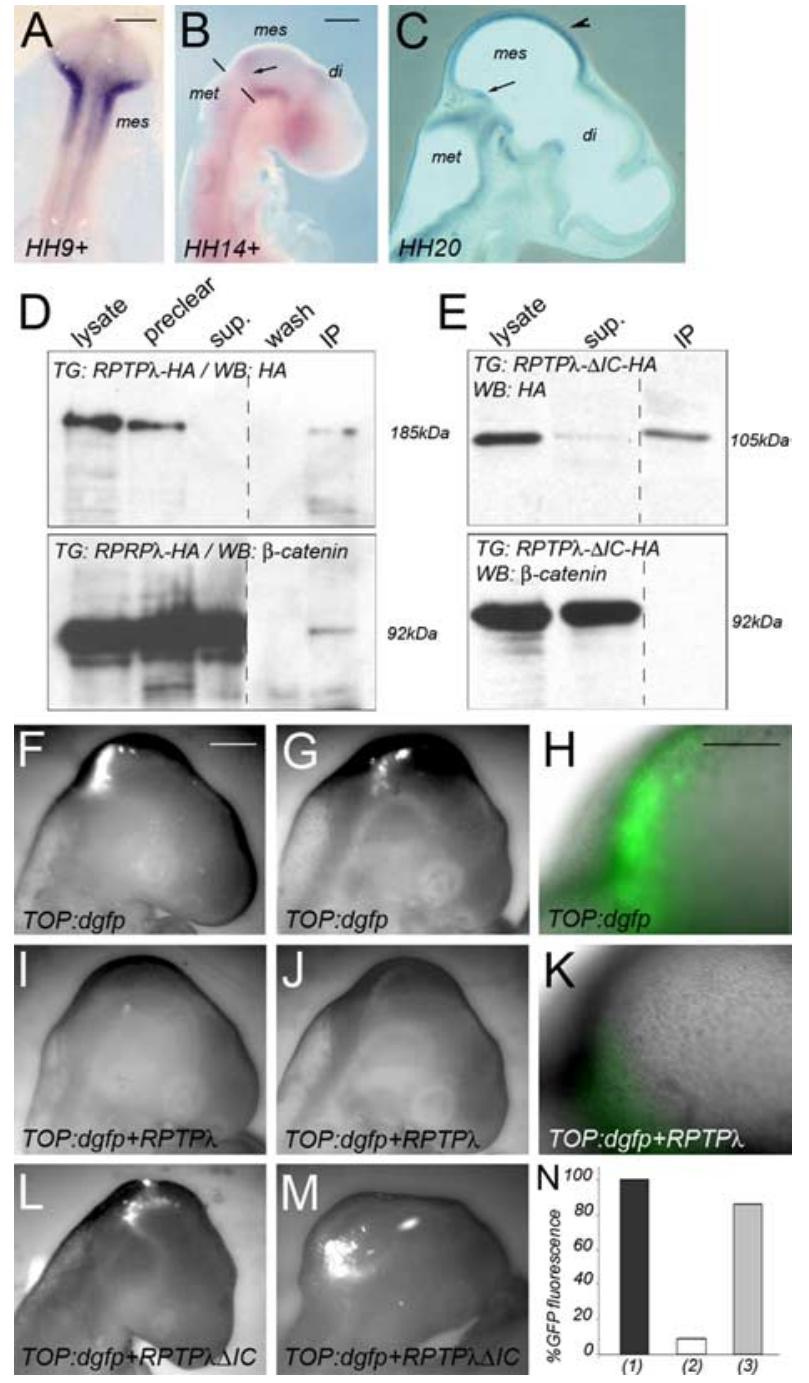

Figure 6. RPTP $\lambda$ binds to $\beta$-catenin in the mesencephalon and inhibits the activity of a $\beta$-catenin responsive promoter in vivo. $\boldsymbol{A}-\boldsymbol{C}, 0 \mathrm{n}$-off-on expression of $R P T P \lambda$ in the mesencephalic vesicle between HH9+ and HH20 as described by Badde et al. (2005). The dashed line in $B$ marks the isthmic constriction, the arrowin $B$ and Cmarks the location of the transversering of $R P T P \lambda$ expression at the MHB, and the arrowhead in C marks the rostral-to-caudal upregulation of RPTP $\lambda$ in the midbrain alar plate. di, Diencephalon; mes, mesencephalon; met, metencephalon. $\boldsymbol{D}$, Immunoprecipitation using an HA-specific antibody on RPTP $\lambda$-HA transfected HH16 midbrain tissue demonstrating coprecipitation of $\beta$-catenin with RPTP $\lambda$-HA. $\boldsymbol{E}$, As in $\boldsymbol{D}$, except tissue transfected with RPTP $\lambda$ $\triangle \mathrm{IC}-\mathrm{HA}$ was used. No coprecipitation with $\beta$-catenin was observed. Top, Western Blot analysis with the HA-specific antibody detecting RPTP $\lambda$-HA (D) or RPTP $\lambda-\Delta \mathrm{IC}-H A(E)$, respectively. Bottom, Detection of $\beta$-catenin on the blots shown above after stripping and reprobing with a $\beta$-catenin-specific antibody. The blots were cut to removethe marker lane. Preclear, Supernatant after precipitation with an unspecific antibody or with agarose-coupled protein-G; sup, supernatant after precipitation with the HA-specific antibody; IP, immunoprecipitate. $\boldsymbol{F - M}$, GFP fluorescence $24 \mathrm{~h}$ after transfection of a TOP:dgfp reporter into the MHB region. $\boldsymbol{F}-\boldsymbol{H}$, Different examples of GFP expression in embryos $24 \mathrm{~h}$ after electroporation with TOP:dgfp. $\boldsymbol{I}-\boldsymbol{K}$, Different examples of GFP expression after electroporation of TOP:dgfp together with RPTP $\lambda$-pMIW. $L, M$, Examples of GFP expression after electroporation of TOP:dgfp together with $R P T P \lambda-\Delta / C$-pMIW. $F, G, I, J, L$, and $M$ are monochrome images. $N$, Quantification of the results: (1), percentage of embryos exhibiting GFP fluorescence $24 \mathrm{~h}$ after transfection with TOP:dgfp alone; (2), percentage of embryos exhibiting GFP fluorescence $24 \mathrm{~h}$ after transfection with TOP:dgfp together with RPTP $\lambda$-HA; (3), percentage of embryos exhibiting GFP fluorescence $24 \mathrm{~h}$ after transfection with TOP:dgfp together with $R P T P \lambda-\Delta / C-H A$. Scale bars: $A, B, 200 \mu \mathrm{m} ; F, 500 \mu \mathrm{m}$; H, $250 \mu \mathrm{m}$.

GFP fluorescence (Fig. $6 L-N)$ ( $n=6$ of 7$)$. These results demonstrate that $R P T P \lambda$ can suppress the activation of this $\beta$-catenin responsive reporter in vivo.

The normal growth and development of the mid-hindbrain
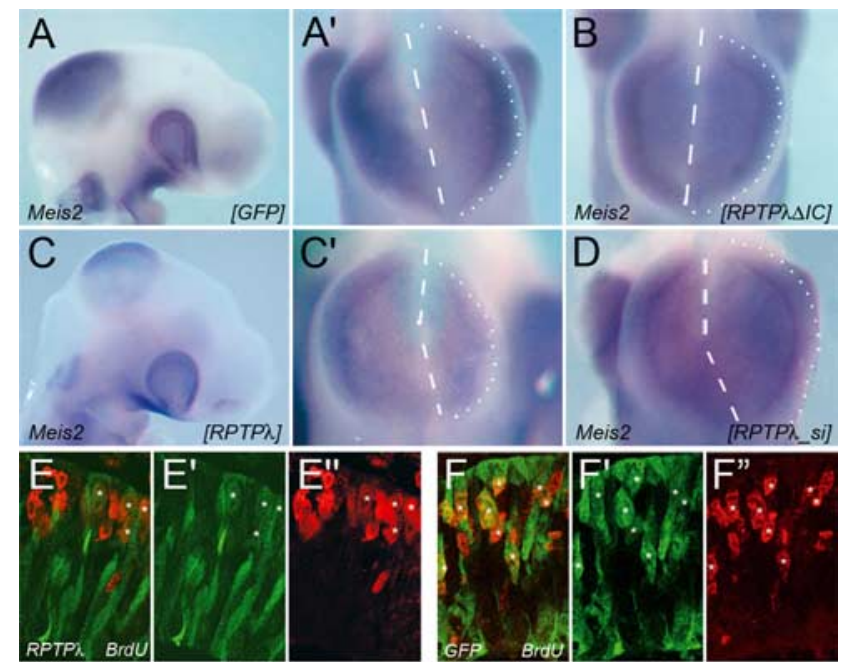

D
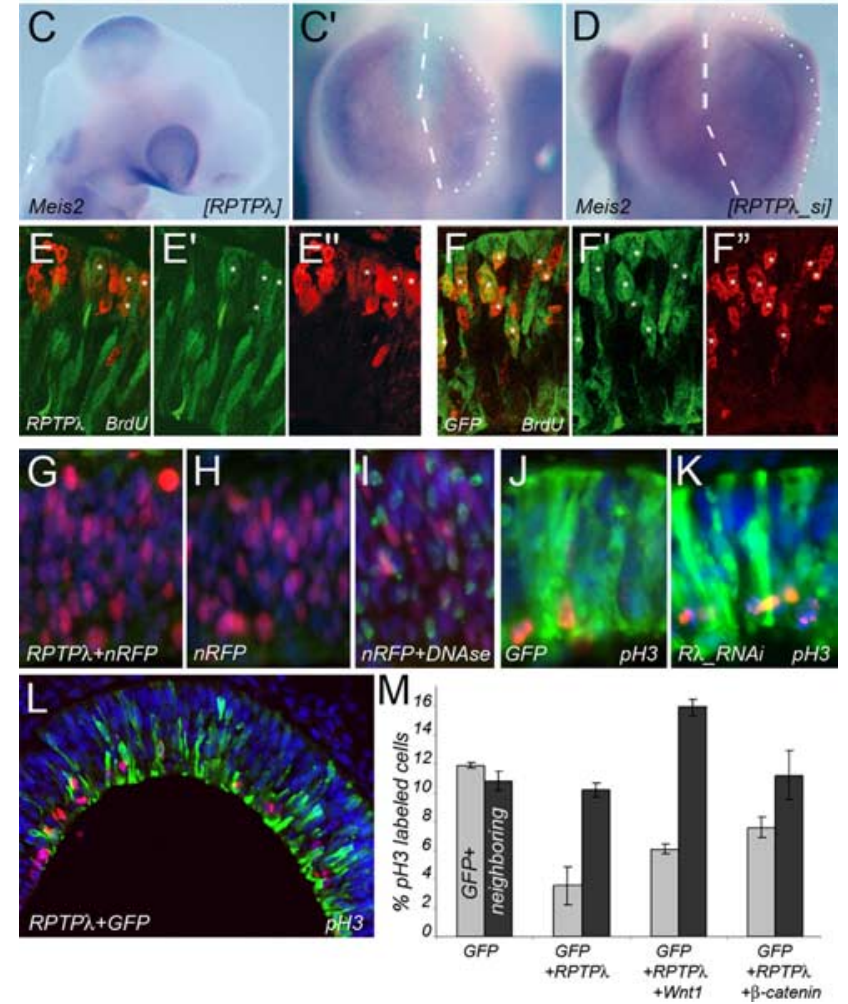

Figure 7. RPTP $\lambda$ modulates mesencephalic growth. $\boldsymbol{A}-\boldsymbol{D}$, Whole-mount in situ hybridization with a Meis2-specific probe on HH15-HH16 embryos after electroporation with pMES ( $A$, $\left.\boldsymbol{A}^{\prime}\right), R P T P \lambda-\Delta / C$-pMES $(\boldsymbol{B}), R P T P \lambda$-pMES $\left(\boldsymbol{C}, \boldsymbol{C}^{\prime}\right)$, or RPTP $\lambda \_$si- $a(\boldsymbol{D})$. In $\boldsymbol{A}^{\prime}, \boldsymbol{B}, \boldsymbol{C}^{\prime}$, and $\boldsymbol{D}$, the embryos are shown from the top. The dashed lines define the location of the dorsal midline as observed by the lack of Meis2 expression, and the dotted lines mark the perimeter of the right side of the mesencephalic vesicle. $\boldsymbol{E}, \boldsymbol{F}$, Cross sections through HH16 midbrains showing BrdU incorporation after ectopic expression of RPTP $\lambda$ together with GFP $\left(\boldsymbol{E}-\boldsymbol{E}^{\prime \prime}\right)$ or GFP alone $\left(\boldsymbol{F}-\boldsymbol{F}^{\prime \prime}\right)$. $\boldsymbol{E}, \boldsymbol{F}$, Overlaid channels of $\boldsymbol{E}^{\prime}$ and $\boldsymbol{E}^{\prime \prime}$ or $\boldsymbol{F}^{\prime}$ and $\boldsymbol{F}^{\prime \prime} . \boldsymbol{E}^{\prime}, \boldsymbol{F}^{\prime}$, GFP expression. $\boldsymbol{E}^{\prime \prime}, \boldsymbol{F}^{\prime \prime}, \operatorname{Brd} \boldsymbol{U}$ incorporation. G-I, TUNEL labeling of HH16 midbrains electroporated with nRFP together with $\operatorname{RPTP} \lambda(\boldsymbol{G})$ or nRFP alone $(\boldsymbol{H})$. $\boldsymbol{I}$, DNase treatment before TUNEL labeling as control for the validity of the experimental procedure. $J, K$, Cross sections through HH16 midbrains labeled for pH3 (red) and GFP (green) and counterstained with DAPI (blue). J, Misexpression of GFP. $\boldsymbol{K}$, Misexpression of RPTP $\lambda \_$si-a together with GFP. L, Cross section through an HH16 midbrain electroporated with RPTP $\lambda$ together with GFP and stained for GFP (green), pH3 (red), and DAPI (blue). Fewer transfected (GFP+) cells are labeled for pH3 than nontransfected, neighboring (GFP-) cells. For more examples, see supplemental Figure 2 (available at www.jneurosci.org as supplemental material).M, Quantification of the percentage of pH3-positive cells transfected with combinations of the gene products indicated (gray bars) and the percentage of $\mathrm{pH} 3$ positive cells among the nontransfected, neighboring cell population under each condition (black bars). Error bars indicate SD. Scale bar $(\boldsymbol{A}), 500 \mu \mathrm{m}$.

region depends on activation of the canonical Wnt signaling pathway (McMahon and Bradley, 1990; Thomas and Capecchi, 1990; Brault et al., 2001; Panhuysen et al., 2004). To investigate whether ectopic $R P T P \lambda$ expression or RNAimediated knock-down of expression altered midbrain growth, $R P T P \lambda$ expression was targeted to the right side of the mesencephalic vesicle by in ovo electroporation into $\mathrm{HH} 9$ embryos, and the consequence of this manipulation on midbrain growth was assessed $24 \mathrm{~h}$ later (Fig. 7). We chose HH9 embryos for these experiments, because this developmental time is before the downregulation of endogenous $R P T P \lambda$ expres- 
sion in the mesencephalon, which occurs after HH12 (Fig. $6 A-C$ ) (Badde et al., 2005). Introduction of $R P T P \lambda$-pMES into the HH9 mesencephalic vesicle therefore maintains $R P T P \lambda$ expression in the midbrain beyond HH12, whereas introduction of an RNAi targeting construct leads to precocious loss of $R P T P \lambda$ expression. The homeodomain Meis 2 was used as a marker for the tectal anlage, because its transcripts are excluded from the roof plate, allowing us to unambiguously determine the location of the dorsal midline and estimate the size of the left and right halves of the mesencephalic vesicle. After forced expression of GFP or of the truncated allele $R P T P \lambda-\Delta I C-H A$ together with GFP, the right and left halves of the tectal anlage appeared to have normal size (Fig. $7 A, B)(n=5$ of 5 for GFP; $n=6$ of 6 for RPTP $\lambda-\Delta I C-H A)$. However, in embryos that had been electroporated with an expression plasmid containing $R P T P \lambda$, the size of the right electroporated tectal anlage was reduced severely when compared with the left, nonelectroporated side of the same embryo (Fig. $\left.7 C, C^{\prime}\right)(n=15$ of 16$)$. Conversely, the right tectal anlage was slightly enlarged in embryos transfected with the specific RNAi targeting construct $R P T P \lambda \_s i-a($ Fig. $7 D)(n=6$ of 7 ).

To determine whether this growth reduction was the result of decreased cell proliferation and/or enhanced cell death, BrdU pulse-labeling experiments were performed. We delivered $R P T P \lambda-H A$ using the electroporation vector pMES, which contains an IRES-GFP cassette allowing us to visualize individual $R P T P \lambda-H A$ transfected cells by their GFP fluorescence (Swartz et al., 2001). Two hours after BrdU injection, $51 \%$ ( \pm 1.85 SEM) of the cells forced to express GFP, but only $27.2 \%$ ( \pm 1.41 SEM; $p<0.002$, Student's $t$ test) of $R P T P \lambda / G F P$ expressing cells had incorporated the BrdU label (Fig. $7 E-F^{\prime \prime}$ ). Similar to mock transfected or nontransfected embryos, apoptotic cell nuclei were rarely observed $24 \mathrm{~h}$ after misexpression of $R P T P \lambda$, indicating that $R P T P \lambda$ electroporation does not compromise cell survival within this time frame (Fig. $7 G-I$ ) $(n=3)$. Knock-down of RPTP $\lambda$ expression at HH9 slightly increased the percentage of mitotic cells, visualized by detecting $\mathrm{pH} 3$ from $11.7 \%( \pm 1.29 \mathrm{SEM})$ after introduction of GFP alone to $14.2 \%$ ( \pm 1.45 SEM) after transfection of the RNAi targeting construct $R P T P \lambda \_s i-a$ together with GFP (Fig. $7 \mathrm{~J}, K$ ) (supplemental Fig. $2 E$, available at www.jneurosci.org as supplemental material) $(n=4)$. In conclusion, these results suggest that RPTP $\lambda$ functions as negative modulator of mesencephalic growth primarily through modulation of progenitor cell proliferation.

Because RPTP $\lambda$ could precipitate (and possibly sequester) the Wnt-signaling intermediate $\beta$-catenin from mesencephalic extracts (Fig. $6 D, E$ ), we wondered whether cotransfection of $\beta$-catenin or $W n t 1$ together with $R P T P \lambda$ would rescue the proliferation defect observed after introduction of $R P T P \lambda$ alone. To test this idea, we introduced either pMES (expressing only GFP), $R P T P \lambda$-pMES (expressing $R P T P \lambda$ together with $G F P), R P T P \lambda$-Wnt1-pMEC together with GFP-pMIW (which results in coexpression of RPTP $\lambda$, Wnt1, and GFP), or $R P T P \lambda$-pMES together with caß-catenin-pMIW (which results in coexpression of $R P T P \lambda, G F P$, and a stabilized form of $\beta$-catenin) into the mesencephalic vesicle at $\mathrm{HH} 9$ and determined the mitotic index of midbrain progenitor cells $24 \mathrm{~h}$ later (Fig. $7 L, M$ ) (supplemental Fig. 2A-D, available at www. jneurosci.org as supplemental material) $(n=9$ for pMES, $R P T P \lambda$-pMES; $n=3$ for RPTP $\lambda$-Wnt 1 -pMEC, $R P T P \lambda$-pMES plus $\beta$-catenin-pMIW). Transfection of $R P T P \lambda$ together with
GFP significantly reduced the percentage of mitotic cells compared with GFP transfected controls. Cotransfection of Wnt1 or stabilized $\beta$-catenin together with RPTP $\lambda$ and GFP partially restored the mitotic index (Fig. $7 M$, gray bars). Therefore, the negative influence of $R P T P \lambda$ on midbrain progenitor cell proliferation could, at least in part, be alleviated by cotransfection of Wnt1 or of the Wnt1-signaling intermediate $\beta$-catenin. To determine whether $R P T P \lambda$ modulates midbrain progenitor cell proliferation in a cell-autonomous or noncellautonomous manner, we compared the percentage of $\mathrm{pH} 3$ positive cell nuclei in the transfected (GFP expressing) cell population under each of these experimental conditions with the percentage of $\mathrm{pH} 3+$ cells in nontransfected (GFPnegative) neighboring cells (Fig. $7 M$, black bars). The mitotic index of cells that were located in close proximity to cells transfected with $R P T P \lambda$ plus GFP did not differ from that of the neighbors of GFP transfected cells, suggesting that RPTP $\lambda$ acts cell autonomously on midbrain progenitor cell proliferation. We also did not observe a significant difference in the percentage of $\mathrm{pH} 3+$ cell nuclei in neighbors of $R P T P \lambda / G F P /$ $\beta$-catenin transfected cells compared with neighbors of GFPexpressing cells. In contrast, the percentage of $\mathrm{pH} 3+$ nuclei in cells located near $R P T P \lambda / W n t 1 / G F P$ transfected cells was clearly increased. This observation is consistent with the notion that the secreted molecule Wnt1 may influence cell proliferation in a cell-nonautonomous manner.

Our finding that overexpression of RPTP $\lambda$ interfered with Fgf8-mediated induction of Wnt1 expression at the MHB as well as with the activation of the $W n t / \beta$-catenin-dependent TOP:dgfp reporter in the mesencephalon raises the question of whether the reduced TOP:dgfp reporter activity in $R P T P \lambda$ transfected embryos may be a secondary consequence of their diminished Wnt1 expression or, as suggested above, may reflect a more direct inhibition of $W n t / \beta$-catenin signaling, presumably through interaction of RPTP $\lambda$ with $\beta$-catenin. To distinguish between these possibilities, we first decreased Wnt1 expression at the MHB through RNAi-mediated knock-down and monitored TOP:dgfp activity in the mesencephalic vesicle $24 \mathrm{~h}$ later (supplemental Fig. $3 A-H^{\prime}$, available at www.jneurosci.org as supplemental material) $(n=$ 15). Although electroporation of Wnt1-specific RNAi targeting constructs reduced $W n t 1$ expression in vivo, GFP expression of the TOP:dgfp reporter appeared normal. Second, we blocked Wnt signaling in the midbrain through forced expression of the known signaling antagonist Axin (Zeng et al., 1997). Wnt1 expression at the MHB was normal $24 \mathrm{~h}$ after transfection (supplemental Fig. 3I,J, available at www. jneurosci.org as supplemental material) $(n=10)$. Based on these observations, we suggest that $R P T P \lambda$ may modulate Wnt1 expression and signal transduction in the midbrain through two separate mechanisms.

The midbrain alar plate undergoes massive expansion between $\mathrm{HH} 12$ and $\mathrm{HH} 19$ when RPTP $\lambda$ expression is absent from this tissue. Cells around the MHB in embryonic day 10.5 (E10.5) to E11.5 mouse embryos are less mitotically active than cells in the midbrain and rhombomere 1 (Trokovic et al., 2005). A similar domain of reduced proliferation also exists at the chick $\mathrm{MHB}$ at HH15 and HH20 (supplemental Fig. 4, available at www. jneurosci.org as supplemental material). Although the ring of $R P T P \lambda$ expression seen during this developmental period does not cover this domain entirely, $R P T P \lambda$ expression nevertheless appears to localize within this domain. 


\section{Discussion}

Here, we present evidence based on gain-of-function and knockdown experiments that $R P T P \lambda$ can counteract transcriptional activation of Wnt1 in the embryonic midbrain. In addition, we show that RPTP $\lambda$ can physically interact with $\beta$-catenin in the midbrain, inhibit the activity of a $\beta$-catenin responsive promoter, and suppress mesencephalic cell proliferation. RPTP $\lambda$ therefore emerges as a potential modulator of Wnt1 expression and signaling at the MHB organizer.

\section{$R P T P \lambda$ is part of the complex molecular interactions that maintain and shape the MHB region}

A complex spatiotemporal pattern of gene expression has been described at the developing mid-hindbrain boundary. The MHB is bordered by a narrow, transverse ring of Wnt 1 expression at its anterior side and by a ring of $F g f 8$ expression at its posterior side. Several proteins, including $P a \times 2 / 5$ and En1/2, are expressed in nested, symmetric gradients across the MHB region. Fgf8 signaling induces activation of the Ras-MAP kinase pathway, which is required for isthmic organizer activity (Sato and Nakamura, 2004). Fgf feedback inhibitors, such as Spry2, Sef, and Mkp3, interfere with Ras-MAP kinase signaling downstream of receptor tyrosine kinases at different steps within the signal transduction pathway and are thought to limit the lateral expansion of the Fgf signal at the MHB (Gross et al., 2001; Nutt et al., 2001; Fürthauer et al., 2002; Lin et al., 2002; Tsang et al., 2002; Kawakami et al., 2003; Echevarria et al., 2005). Maximal Ras-MAP kinase activation occurs within the domain of $F g f 8$ expression but tapers off into the mesencephalic and metencephalic vesicles (Corson et al., 2003; Sato and Nakamura, 2004). The shapes of the Pax2/5 and En1/2 expression domains reflect the gradient of Ras-MAP kinase activity at the MHB. In contrast, Wnt1 expression is confined to a tight ring of cells in the mesencephalon and is absent from the metencephalon. Metencephalic tissue is not permissive for Wnt1 expression, explaining the asymmetric nature of the Wnt1 expression domain (Kikuta et al., 2003). It is yet unclear, however, as to why Wnt1 expression is restricted to such a discrete band of cells and does not, like that of Pax2/5 or En1/2, taper off into the mesencephalic vesicle. As we show here, the receptor tyrosine phosphatase $\lambda$ is expressed in a ring of cells rostral to the Wnt1 domain and inhibits Wnt1 expression when overexpressed. $R P T P \lambda$ thus has the potential to restrict Wnt1 expression to its characteristic tight ring at the MHB.

$R P T P \lambda$ expression itself is induced by $F g f 8 b$ in a concentration-dependent manner. When Fgf8-induced expression of $W n t 1$ and RPTP $\lambda$ was monitored successively in the same specimen, ectopic expression of $R P T P \lambda$ could always be observed anterior to the ring of $W n t 1$ expression. Induction of $W n t 1$ and $R P T P \lambda$ expression by Fgf 8 thus mimics their normal spatial relationship at the MHB, where the $W n t 1$ expression also separates the domain of $R P T P \lambda$ expression from that of $F g f 8$. In addition, after implantation of Fgf8b releasing beads, ectopic $R P T P \lambda$ expression could be observed at a Fgf8b concentration that was not sufficient to induce Wnt1 expression. We conclude from these results that different dosages of the Fgf8 signal from the MHB are required for transcriptional induction of $R P T P \lambda$ and Wnt1, respectively. Examples for dosage dependency of Fgf function during embryogenesis have been described previously and include telencephalic and cerebellar development, patterning of the foregut, and skeletal development (Sato et al., 2001; Storm et al., 2003, 2006; Hajihosseini et al., 2004).

$R P T P \lambda$ specifically inhibited Wnt1 expression but not that of other MHB marker genes when overexpressed in the MHB re- gion. In addition, when cells in the midbrain were forced to express $R P T P \lambda$, they failed to upregulate $W n t 1$ expression after subsequent stimulation by $F g f 8$, yet they readily expressed $E n 1$, Pax2, or Pax5 under identical experimental conditions. RPTP $\lambda$ therefore appears to specifically interfere with $F g f 8$-induced transcriptional activation of Wnt1 at the MHB. How can this high degree of functional specificity be achieved mechanistically? Expression of many MHB marker genes, including Otx2, Gbx2, $P a x 2 / 5, E n 1 / 2$, and $W n t 1$, is negatively affected by overexpression of a dominant-negative form of Ras (Sato and Nakamura, 2004). Yet, we observed recently after transfection of different concentrations of a constitutive active form of Ras (caRas) that transcriptional activation of Wnt 1 at the MHB could only be induced at a narrow concentration range of $c a R a s$, whereas transcriptional activation of $E n 1$ was observed over a concentration range spanning more than an order of magnitude (Vennemann et al., 2008). Induction of Wnt1 at the organizer thus appears to be more sensitive to changes in the strength of Ras-MAP kinase pathway activation than other MHB marker genes. This raises the possibility that tight, local regulation of Ras-MAP kinase activity may be a prerequisite for Wnt1 expression at the $\mathrm{MHB}$ and that $R P T P \lambda$ may contribute to this regulation through modulation of Ras-MAP kinase signaling. Notably, in mouse embryos in which Fgf8 expression was specifically lost in the MHB territory from the five somite stage onwards, the transverse ring of $W n t 1$ expression in the caudal mesencephalon was also absent, whereas the expression domains of Fgf8, Otx2, Pax2, or En1 appeared normal at early stages of embryogenesis (Chi et al., 2003). Genetic loss of $F g f 8$ at the MHB, however, caused massive cell death, an effect we did not observe within $24 \mathrm{~h}$ after $R P T P \lambda$ transfection. We cannot rule out that $R P T P \lambda$ inhibits $W n t 1$ expression by interfering with still unknown factor(s) that are specifically required for transcriptional Wnt1 activation in Fgf8 responding cells. Regardless of the exact molecular nature of this inhibition, the experiments described here suggest a model in which Fgf8 induces $W n t 1$ and $R P T P \lambda$ expression in the midbrain at different signaling thresholds and consequently at different distances to the MHB, so that the Wnt1 domain is always bordered by $R P T P \lambda$-expressing cells at its rostral side. One function of $R P T P \lambda$ at the MHB may thus be to act as a Ras-MAP kinase feedback inhibitor to restrict the anterior expansion of the transverse Wnt1 domain (Fig. 8).

\section{RPTP $\lambda$ binds to $\beta$-catenin and modulates canonical Wnt1 signaling at the MHB}

In addition to restricting $W n t 1$ expression at the MHB, RPTP $\lambda$ also seems to play a role in modulating $W n t 1$ signal transduction. In the canonical or $W n t / \beta$-catenin pathway, $W n t$ binding to frizzled receptors and $L R P$ coreceptors inhibits degradation of the cytoplasmic pool of $\beta$-catenin, allowing it to translocate to the nucleus, where it acts as coactivator for $L E F / T C F$ transcription factors to promote target gene expression (Logan and Nusse, 2004). In addition to its role in Wnt signaling, $\beta$-catenin is a structural link between cadherins/ $\alpha$-catenin and the actin cytoskeleton. Cadherin bound $\beta$-catenin can be freed to participate in Wnt signaling and vice versa, a switch that appears to be regulated by phosphorylation of $\beta$-catenin on tyrosine residues (Brembeck et al., 2004; Nelson and Nusse, 2004; Schambony et al., 2004; Lilien and Balsamo, 2005). It was shown previously that $\beta$-catenin interacts with the juxtamembrane domain of the human homolog of $R P T P \lambda$ and can be dephosphorylated by this protein in vitro (Yan et al., 2002). As shown here, a similar interaction of $R P T P \lambda$ and 


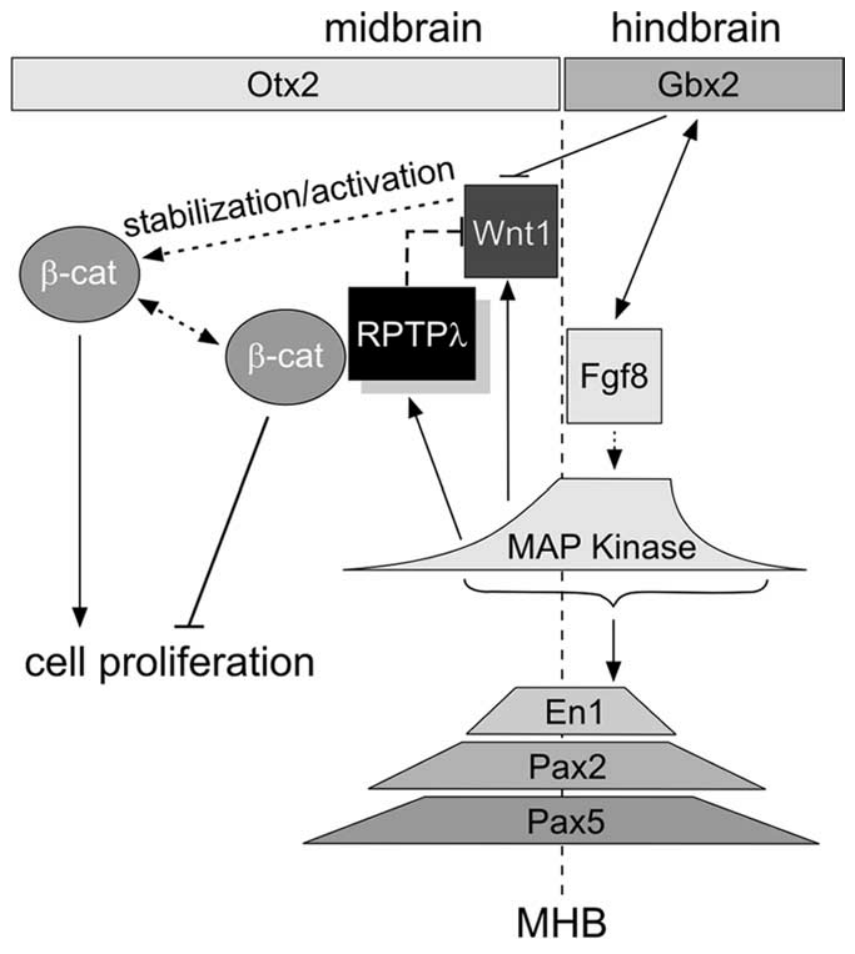

Figure 8. Proposed model for the function of RPTP $\lambda$ at the MHB. The MHB (dashed vertical line) is located at the border between the expression domains of 0 tx2 (rostral) and Gbx2 (caudal). Once the MHB is established during early somitogenesis, Fgf8, Wnt1, En2, Pax2, and Pax5 function in an interdependent, positive feedback loop, which is necessary for organizer maintenance. RPTP $\lambda$ expression is induced by Fgf8 at a signaling threshold that differs from that required for Wnt 1 induction, resulting in a band of RPTP $\lambda$ expression anterior to the Wnt1 domain. RPTP $\lambda$ counteracts Fgf8-mediated transcriptional activation of Wnt1, which constrains the Wnt1 domain to its typical tight ring anterior to the $0 t \times 2-G b \times 2$ boundary. Presumably, as a consequence of the high degree of homology between the juxtamembrane domain of RPTP $\lambda$ with cadherins, RPTP $\lambda$ can bind to and sequester $\beta$-catenin and thereby negatively influence canonical Wnt signaling.

$\beta$-catenin also occurs in the embryonic chick midbrain. In addition, when full-length $R P T P \lambda$ was overexpressed, we found that two functional readouts of canonical Wnt signaling in the midbrain, activation of a $\beta$-catenin responsive promoter and neuroepithelial cell proliferation, were reduced. This proliferation defect could be partially reversed by cotransfection of Wnt 1 or of a stabilized form of $\beta$-catenin. It is intriguing to speculate that $R P T P \lambda$ may antagonize canonical Wnt signaling through binding and potentially dephosphorylation of $\beta$-catenin and ultimately serve as a negative regulator of cell proliferation in the developing midbrain (Fig. 8). Decreasing Wnt1 expression at the MHB through transfection of Wnt1specific RNAi targeting constructs did not notably reduce $\beta$-catenin responsive reporter activity in the midbrain, nor did misexpression of Axin, a known inhibitor of the Wnt signaling pathway, in the mesencephalon disturb the transverse ring of Wnt1 expression at the organizer. These observations suggest that $R P T P \lambda$ acts on Wnt1 expression and signal transduction through two independent mechanisms.

How can this dual effect be explained? Protein tyrosine phosphatases antagonize the activity of protein tyrosine kinases and influence a broad spectrum of physiological processes. For instance, $R P T P \lambda$ (also termed RPTP $\psi$ ) is required for Delta/Notchdependent oscillatory gene expression in the presomitic mesoderm as well as for convergent extension during gastrulation through an as yet unknown mechanism (Aerne and Ish-
Horowicz, 2004). It is therefore possible that $R P T P \lambda$ impinges on multiple signaling pathways through dephosphorylation of different signaling components. In addition, the ring of $R P T P \lambda$ expression at the MHB may coincide with that of another receptor tyrosine phosphatase, $R P T P \zeta / \beta$, a keratan sulfate modified protein, which has been suggested to modify signal transduction at the MHB through influencing the diffusion of morphogenetic signals of the Wnt and Fgf families (Canoll et al., 1993; Hamanaka et al., 1997). Although the precise role of RPTP $\lambda$ in any of these processes will only be fully understood once its substrates are identified, our results suggest that $R P T P \lambda$ is part of the complex molecular network that governs development of the midbrain region.

\section{References}

Adams KA, Maida JM, Golden JA, Riddle RD (2000) The transcription factor Lmxlb maintains Wnt1 expression within the isthmic organizer. Development 127:1857-1867.

Aerne B, Ish-Horowicz D (2004) Receptor tyrosine phosphatase psi is required for Delta/Notch signalling and cyclic gene expression in the presomitic mesoderm. Development 131:3391-3399.

Aerne B, Stoker A, Ish-Horowicz D (2003) Chick receptor tyrosine phosphatase Psi is dynamically expressed during somitogenesis. Gene Expr Patterns 3:325-329.

Araki I, Nakamura H (1999) Engrailed defines the position of dorsal dimesencephalic boundary by repressing diencephalic fate. Development 126:5127-5135.

Badde A, Bumsted-O'Brien KM, Schulte D (2005) Chick receptor protein tyrosine phosphatase lambda/psi (cRPTPlambda/cRPTPpsi) is dynamically expressed at the midbrain-hindbrain boundary and in the embryonic neural retina. Gene Expr Patterns 5:786-791.

Bally-Cuif L, Alvarado-Mallart RM, Darnell DK, Wassef M (1992) Relationship between Wnt-1 and En-2 expression domains during early development of normal and ectopic met-mesencephalon. Development 115:999-1009.

Bally-Cuif L, Cholley B, Wassef M (1995) Involvement of Wnt-1 in the formation of the mes/metencephalic boundary. Mech Dev 53:23-34.

Brault V, Moore R, Kutsch S, Ishibashi M, Rowitch DH, McMahon AP, Sommer L, Boussadia O, Kemler R (2001) Inactivation of the betacatenin gene by Wnt1-Cre-mediated deletion results in dramatic brain malformation and failure of craniofacial development. Development 128:1253-1264.

Brembeck FH, Schwarz-Romond T, Bakkers J, Wilhelm S, Hammerschmidt M, Birchmeier W (2004) Essential role of BCL9-2 in the switch between beta-catenin's adhesive and transcriptional functions. Genes Dev 18:2225-2230.

Canoll PD, Barnea G, Levy JB, Sap J, Ehrlich M, Silvennoinen O, Schlessinger J, Musacchio JM (1993) The expression of a novel receptor-type tyrosine phosphatase suggests a role in morphogenesis and plasticity of the nervous system. Dev Brain Res 75:293-298.

Chi CL, Martinez S, Wurst W, Martin GR (2003) The isthmic organizer signal Fgf8 is required for cell survival in the prospective midbrain and cerebellum. Development 130:2633-2644.

Corson LB, Yamanaka Y, Lai KM, Rossant J (2003) Spatial and temporal patterns of ERK signaling during mouse embryogenesis. Development 130:4527-4537.

Crossley PH, Martinez S, Martin GR (1996) Midbrain development induced by FGF8 in the chick embryo. Nature 380:66-68.

Dailey L, Ambrosetti D, Mansukhani A, Basilico C (2005) Mechanisms underlying differential responses to FGF signaling. Cytokine Growth Factor Rev 16:233-247.

den Hertog J (1999) Protein-tyrosine phosphatases in development. Mech Dev 85:3-14.

Dorsky RI, Sheldahl LC, Moon RT (2002) A transgenic Lef1/beta-catenindependent reporter is expressed in spatially restricted domains throughout zebrafish development. Dev Biol 241:229-237.

Echevarria D, Martinez S, Marques S, Lucas-Teixeira V, Belo JA (2005) Mkp3 is a negative feedback modulator of Fgf8 signaling in the mammalian isthmic organizer. Dev Biol 277:114-128.

Funayama N, Fagotto F, McCrea P, Gumbiner BM (1995) Embryonic axis 
induction by the armadillo repeat domain of beta-catenin: evidence for intracellular signaling. J Cell Biol 128:959-968.

Fürthauer M, Lin W, Ang SL, Thisse B, Thisse C (2002) Sef is a feedbackinduced antagonist of Ras/MAPK-mediated FGF signalling. Nat Cell Biol 4:170-174.

Gross I, Bassit B, Benezra M, Licht JD (2001) Mammalian sprouty proteins inhibit cell growth and differentiation by preventing ras activation. J Biol Chem 276:46460-46468.

Hajihosseini MK, Lalioti MD, Arthaud S, Burgar HR, Brown JM, Twigg SR, Wilkie AO, Heath JK (2004) Skeletal development is regulated by fibroblast growth factor receptor 1 signalling dynamics. Development 131:325-335.

Hamanaka H, Maeda N, Noda M (1997) Spatially and temporally regulated modification of the receptor-like protein tyrosine phsophatase $\zeta / \beta$ isoforms with keratan sulphate in the developing chick brain. Eur J Neurosci 9:2297-2308.

Hamburger V, Hamilton HL (1992) A series of normal stages in the development of the chick embryo. (1951). Dev Dyn 195:231-272.

Hidalgo-Sánchez M, Martínez-de-la-Torre M, Alvarado-Mallart RM, Puelles L (2005) A distinct preisthmic hitogenetic domain is defined by overlap of Otx2 and Pax2 gene expression in the avian caudal midbrain. J Comp Neurol 483:17-29.

Hirata H, Tomita K, Bessho Y, Kageyama R (2001) Hes1 and Hes3 regulate maintenance of the isthmic organizer and development of the mid/hindbrain. EMBO J 20:4454-4466.

Hollyday M, McMahon JA, McMahon AP (1995) Wnt expression patterns in chick embryo nervous system. Mech Dev 52:9-25.

Irving C, Mason I (2000) Signalling by FGF8 from the isthmus patterns anterior hindbrain and establishes the anterior limit of Hox gene expression. Development 127:177-186.

Itoh M, Kudoh T, Dedekian M, Kim CH, Chitnis AB (2002) A role for irol and iro7 in the establishment of an anteroposterior compartment of the ectoderm adjacent to the midbrain-hindbrain boundary. Development 129:2317-2327.

Kawakami Y, Rodríguez-León J, Koth CM, Büscher D, Itoh T, Raya A, Ng JK, Esteban CR, Takahashi S, Henrique D, Schwarz MF, Asahara H, Izpisúa Belmonte JC (2003) MKP3 mediates the cellular response to FGF8 signalling in the vertebrate limb. Nat Cell Biol 5:513-519.

Kikuta H, Kanai M, Ito Y, Yamasu K (2003) gbx2 Homeobox gene is required for the maintenance of the isthmic region in the zebrafish embryonic brain. Dev Dyn 228:433-450.

Korinek V, Barker N, Morin PJ, van Wichen D, de Weger R, Kinzler KW, Vogelstein B, Clevers H (1997) Constitutive transcriptional activation by a beta-catenin-Tcf complex in APC-1- colon carcinoma. Science 275:1784-1787.

Lilien J, Balsamo J (2005) The regulation of cadherin-mediated adhesion by tyrosine phosphorylation/dephosphorylation of beta-catenin. Curr Opin Cell Biol 17:459-465.

Lin W, Fürthauer M, Thisse B, Thisse C, Jing N, Ang SL (2002) Cloning of the mouse Sef gene and comparative analysis of its expression with Fgf8 and Spry2 during embryogenesis. Mech Dev 113:163-168.

Logan CY, Nusse R (2004) The Wnt signaling pathway in development and disease. Annu Rev Cell Dev Biol 20:781-810.

Martinez S, Alvarado-Mallart RM (1990) Expression of the homeobox Chick-en gene in chick/quail chimeras with inverted mes-metencephalic grafts. Dev Biol 139:432-436.

Martinez S, Wassef M, Alvarado-Mallart RM (1991) Induction of a mesencephalic phenotype in the 2-day-old chick prosencephalon is preceded by the early expression of the homeobox gene en. Neuron 6:971-981.

Martinez S, Crossley PH, Cobos I, Rubenstein JL, Martin GR (1999) FGF8 induces formation of an ectopic isthmic organizer and isthmocerebellar development via a repressive effect on Otx2 expression. Development 126:1189-1200.

Matsunaga E, Katahira T, Nakamura H (2002) Role of Lmx1b and Wnt1 in mesencephalon and metencephalon development. Development 129:5269-5277.

McMahon AP, Bradley A (1990) The Wnt-1 (int-1) proto-oncogene is required for development of a large region of the mouse brain. Cell 62:1073-1085.

McMahon AP, Joyner AL, Bradley A, McMahon JA (1992) The midbrainhindbrain phenotype of Wnt-1-/Wnt-1 - mice results from stepwise dele- tion of engrailed-expressing cells by 9.5 days postcoitum. Cell 69:581-595.

Meyers EN, Lewandoski M, Martin GR (1998) An Fgf8 mutant allelic series generated by Cre- and Flp-mediated recombination. Nat Genet 18:136-141.

Momose T, Tonegawa A, Takeuchi J, Ogawa H, Umesono K, Yasuda K (1999) Efficient targeting of gene expression in chick embryos by microelectroporation. Dev Growth Differ 41:335-344.

Mühleisen TW, Agoston Z, Schulte D (2006) Retroviral misexpression of cVax disturbs retinal ganglion cell axon fasciculation and intraretinal pathfinding in vivo and guidance of nasal ganglion cell axons in vivo. Dev Biol 297:59-73.

Nakamura H, Nakano KE, Igawa HH, Takagi S, Fujisawa H (1986) Plasticity and rigidity of differentiation of brain vesicles studied in quail-chick chimeras. Cell Differ 19:187-193.

Nelson WJ, Nusse R (2004) Convergence of Wnt, beta-catenin, and cadherin pathways. Science 303:1483-1487.

Nutt SL, Dingwell KS, Holt CE, Amaya E (2001) Xenopus Sprouty2 inhibits FGF-mediated gastrulation movements but does not affect mesoderm induction and patterning. Genes Dev 15:1152-1166.

O’Hara FP, Beck E, Barr LK, Wong LL, Kessler DS, Riddle RD (2005) Zebrafish Lmxlb.1 and Lmx1b.2 are required for maintenance of the isthmic organizer. Development 132:3163-3173.

Orsulic S, Huber O, Aberle H, Arnold S, Kemler R (1999) E-cadherin binding prevents beta-catenin nuclear localization and beta-catenin/LEF-1mediated transactivation. J Cell Sci 112:1237-1245.

Panhuysen M, Vogt Weisenhorn DM, Blanquet V, Brodski C, Heinzmann U, Beisker W, Wurst W (2004) Effects of Wnt1 signaling on proliferation in the developing mid-/hindbrain region. Mol Cell Neurosci 26:101-111.

Paul S, Lombroso PJ (2003) Receptor and nonreceptor protein tyrosine phosphatases in the nervous system. Cell Mol Life Sci 60:2465-2482.

Reifers F, Böhli H, Walsh EC, Crossley PH, Stainier DY, Brand M (1998) Fgf8 is mutated in zebrafish acerebellar (ace) mutants and is required for maintenance of midbrain-hindbrain boundary development and somitogenesis. Development 125:2381-2395.

Rowitch DH, McMahon AP (1995) Pax-2 expression in the murine neural plate precedes and encompasses the expression domains of Wnt- 1 and En-1. Mech Dev 52:3-8.

Sato T, Nakamura H (2004) The Fgf8 signal causes cerebellar differentiation by activating the Ras-ERK signaling pathway. Development 131:4275-4285.

Sato T, Araki I, Nakamura H (2001) Inductive signal and tissue responsiveness defining the tectum and the cerebellum. Development 128:2461-2469.

Schambony A, Kunz M, Gradl D (2004) Cross-regulation of Wnt signaling and cell adhesion. Differentiation 72:307-318.

Schulte D, Cepko CL (2000) Two homeobox genes define the domain of EphA3 expression in the developing chick retina. Development 127:5033-5045.

Schulte D, Furukawa T, Peters MA, Kozak CA, Cepko CL (1999) Misexpression of the Emx-related homeobox genes cVax and mVax2 ventralizes the retina and perturbs the retinotectal map. Neuron 24:541-553.

Shamim H, Mahmood R, Logan C, Doherty P, Lumsden A, Mason I (1999) Sequential roles for Fgf4, En1 and Fgf8 in specification and regionalisation of the midbrain. Development 126:945-959.

Stockinger A, Eger A, Wolf J, Beug H, Foisner R (2001) E-cadherin regulates cell growth by modulating proliferation-dependent beta-catenin transcriptional activity. J Cell Biol 154:1185-1196.

Stoker A, Dutta R (1998) Protein tyrosine phosphatases and neural development. BioEssays 20:463-472.

Storm EE, Rubenstein JL, Martin GR (2003) Dosage of Fgf8 determines whether cell survival is positively or negatively regulated in the developing forebrain. Proc Natl Acad Sci USA 100:1757-1762.

Storm EE, Garel S, Borello U, Hebert JM, Martinez S, McConnell SK, Martin GR, Rubenstein JL (2006) Dose-dependent functions of Fgf8 in regulating telencephalic patterning centers. Development 133:1831-1844.

Suemori H, Kadodawa Y, Goto K, Araki I, Kondoh H, Nakatsuji N (1990) A mouse embryonic stem cell line showing pluripotency of differentiation in early embryos and ubiquitous beta-galactosidase expression. Cell Differ Dev 29:181-186. 
Swartz ME, Eberhart J, Pasquale EB, Krull CE (2001) EphA4/ephrin-A5 interactions in muscle precursor cell migration in the avian forelimb. Development 128:4669-4680.

Thomas KR, Capecchi MR (1990) Targeted disruption of the murine int-1 proto-oncogene resulting in severe abnormalities in midbrain and cerebellar development. Nature 346:847-850.

Tonks NK (2006) Protein tyrosine phosphatases: from genes, to function, to disease. Nat Rev Mol Cell Biol 7:833-846.

Trokovic R, Trokovic N, Hernesniemi S, Pirvola U, Vogt Weisenhorn DM, Rossant J, McMahon AP, Wurst W, Partanen J (2003) FGFR1 is independently required in both developing mid- and hindbrain for sustained response to isthmic signals. EMBO J 22:1811-1823.

Trokovic R, Jukkola T, Saarimäki J, Peltopuro P, Naserke T, Weisenhorn DM, Trokovic N, Wurst W, Partanen J (2005) Fgfrl-dependent boundary cells between developing mid- and hindbrain. Dev Biol 278:428-439.
Tsang M, Friesel R, Kudoh T, Dawid IB (2002) Identification of Sef, a novel modulator of FGF signalling. Nat Cell Biol 4:165-169.

Vennemann A, Agoston Z, Schulte D (2008) Differential and dosedependent regulation of gene expression at the mid-hindbrain boundary by Ras-MAP kinase signaling. Brain Res 1206:33-43.

Wang H, Lian Z, Lerch MM, Chen Z, Xie W, Ullrich A (1996) Characterization of PCP-2, a novel receptor protein tyrosine phosphatase of the MAM domain family. Oncogene 12:2555-2562.

Yan HX, He YQ, Dong H, Zhang P, Zeng JZ, Cao HF, Wu MC, Wang HY (2002) Physical and functional interaction between receptor-like protein tyrosine phosphatase PCP-2 and beta-catenin. Biochemistry 41:15854-15860.

Zeng L, Fagotto F, Zhang T, Hsu W, Vasicek TJ, Perry III WL, Lee JJ, Tilghman SM, Gumbiner BM, Costantini F (1997) The mouse Fused locus encodes Axin, an inhibitor of the Wnt signaling pathway that regulates embryonic axis formation. Cell 90:181-192. 